\title{
CaMKII Controls Whether Touch Is Painful
}

\author{
Hongwei Yu, ${ }^{1}$ Bin Pan, ${ }^{1}$ Andy Weyer, ${ }^{2}$ Hsiang-En Wu,${ }^{1}$ Jingwei Meng, ${ }^{3}$ Gregory Fischer, ${ }^{1}$ Daniel Vilceanu, ${ }^{1}$ \\ Alan R. Light, ${ }^{4}$ Cheryl Stucky, ${ }^{2}$ Frank L. Rice, ${ }^{5}$ Andy Hudmon, ${ }^{3}$ and Quinn Hogan ${ }^{1,6}$ \\ ${ }^{1}$ Department of Anesthesiology and ${ }^{2}$ Department of Cell Biology, Neurobiology, and Anatomy, Medical College of Wisconsin, Milwaukee, Wisconsin 53226, \\ ${ }^{3}$ Department of Biochemistry and Molecular Biology, Stark Neurosciences Research Institute, Indiana University School of Medicine, Indianapolis, Indiana \\ 46202, ${ }^{4}$ Departments of Anesthesiology, and Neurobiology and Anatomy, University of Utah School of Medicine, Salt Lake City, Utah 84132, ${ }^{5}$ Integrated \\ Tissue Dynamics LLC, Rensselaer, New York 12144 and Department of Biomedical Sciences, State University of New York at Albany, Albany, New York \\ 12222, and ${ }^{6}$ Zablocki Veterans Administration Medical Center, Milwaukee, Wisconsin 53295
}

The sensation of touch is initiated when fast conducting low-threshold mechanoreceptors (A $\beta$-LTMRs) generate impulses at their terminals in the skin. Plasticity in this system is evident in the process of adaption, in which a period of diminished sensitivity follows prior stimulation. CaMKII is an ideal candidate for mediating activity-dependent plasticity in touch because it shifts into an enhanced activation state after neuronal depolarizations and can thereby reflect past firing history. Here we show that sensory neuron CaMKII autophosphorylation encodes the level of $A \beta$-LTMR activity in rat models of sensory deprivation (whisker clipping, tail suspension, casting). Blockade of CaMKII signaling limits normal adaptation of action potential generation in A $\beta$-LTMRs in excised skin. CaMKII activity is also required for natural filtering of impulse trains as they travel through the sensory neuron T-junction in the DRG. Blockade of CaMKII selectively in presynaptic $\mathrm{A} \beta$-LTMRs removes dorsal horn inhibition that otherwise prevents A $\beta$-LTMR input from activating nociceptive lamina I neurons. Together, these consequences of reduced CaMKII function in A $\beta$-LTMRs cause low-intensity mechanical stimulation to produce pain behavior. We conclude that, without normal sensory activity to maintain adequate levels of CaMKII function, the touch pathway shifts into a pain system. In the clinical setting, sensory disuse may be a critical factor that enhances and prolongs chronic pain initiated by other conditions.

Key words: adaptation; CaMKII; DRG; mechanosensation; pain; sensory neuron

\section{Significance Statement}

The sensation of touch is served by specialized sensory neurons termed low-threshold mechanoreceptors (LTMRs). We examined the role of CaMKII in regulating the function of these neurons. Loss of CaMKII function, such as occurred in rats during sensory deprivation, elevated the generation and propagation of impulses by LTMRs, and altered the spinal cord circuitry in such a way that low-threshold mechanical stimuli produced pain behavior. Because limbs are protected from use during a painful condition, this sensitization of LTMRs may perpetuate pain and prevent functional rehabilitation.

\section{Introduction}

The mammalian nervous system has evolved the ability to process external information using various modalities of sensory re-

\footnotetext{
Received May 21, 2015; revised Aug. 13, 2015; accepted Aug. 14, 2015.

Author contributions: H.Y., B.P., A.H., and Q.H. designed research; H.Y., B.P., A.W., H.-E.W., J.M., G.F., D.V., C.S., F.L.R., A.H., and Q.H. performed research; H.Y., B.P., A.W., H.-E.W., G.F., D.V., A.R.L., C.S., F.L.R., A.H., and Q.H. analyzed data; H.Y., B.P., A.R.L., F.L.R., A.H., and Q.H. wrote the paper.

This work was supported by National Institutes of Health Grant NS042150 and Veterans Administration Merit Review Grant RX000586 to Q.H., Advancing a Healthier Wisconsin Research and Education Grant 5520293 to Q.H. and C.S., and National Institutes of Health Grant NS078171 and Indiana State Department of Health Grant ISDH/A702-079607 to A.H. We thank Dr. Steven H. Green (Department of Biological Sciences, University of lowa) for providing the EGFP-AIP plasmid.

The authors declare no competing financial interests.

Correspondence should be addressed to Dr. Quinn Hogan, Department of Anesthesiology, Medical College of Wisconsin, 8701 West Watertown Plank Road, Milwaukee, WI 53226-3548. E-mail: qhogan@mcw.edu. DOI:10.1523/JNEUROSCI.1969-15.2015

Copyright $\odot 2015$ the authors $\quad 0270-6474 / 15 / 3514086-17 \$ 15.00 / 0$
}

ceptors, neural connections, and specific elements in the brain that ultimately decode the peripheral and central elements into a sensory perception. A fundamental feature of sensory systems is adaption: the ability to respond to sustained shifts in stimulation intensity by compensatory adjustments in responsiveness of the neuronal pathways. This functional plasticity extends the perceptive range while optimizing signal detection and discrimination. The underlying mechanisms contributing to adaptation have been less explored for the sense of touch than for the visual, olfactory, auditory, and proprioceptive systems (Torre et al., 1995). However, it is clear that touch is subject to use-dependent sensory adaptation because cutaneous mechanical hypersensitivity develops in the absence of normal sensory activation in rats (Guo et al., 2004) and human subjects (Aftanas and Zubek, 1963; Terkelsen et al., 2008), and reduced sensitivity follows prolonged cutaneous stimulation (Aftanas and Zubek, 1963). 


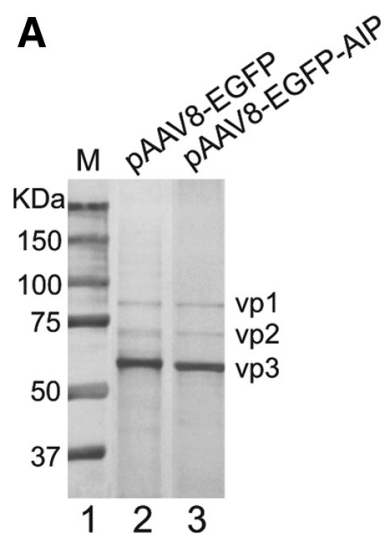

B
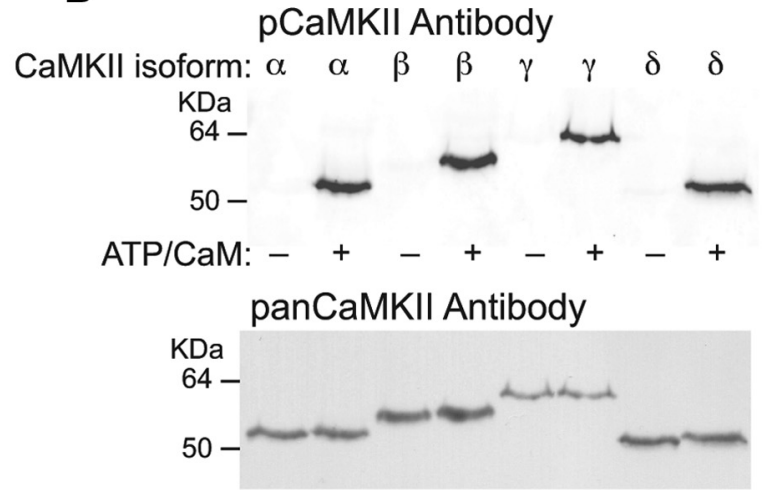

Figure 1. Vector and antibody validation. $A$, Silver staining of SDS-PAGE gel loaded with $5 \times 10^{9}$ viral particles for AAV8-EGFP (lane 2) and AAV8-EGFP-AIP (lane 3) revealed the correct ratios of 1:1:10 for Vp1, Vp2, and Vp3 of AAV virion capsid proteins. Combined, these accounted for $95.9 \%$ of total silver-stained proteins for AAV8-EGFP and $95.2 \%$ of total silver-stained proteins for AAV8-EGFP-AIP, demonstrating high purity. M, Marker, lane 1. B, Western blot shows that the pCaMKII antibody (Santa Cruz Biotechnology, \#12886r) used to quantify CaMKIII Thr286/287 autophosphorylation recognizes all four purified CaMKII isoforms. This phospho-antibody recognizes only CaMKII, the phosphorylated/activated state (upper Western). Bottom panel, Same membrane probed with a panCaMKII antibody (Epitomics, \#2048-1).

Basic mechanisms leading to sensory adaptation are found along the entire sensory pathway from peripheral transduction to higher brain levels (Webster, 2012). The rapidly conducting (A $\beta$ ) low-threshold mechanoreceptors (LTMRs), which are the peripheral sensory neurons serving the sense of touch, show usedependent plasticity in single-unit recordings in which firing rates decline over the first few seconds of a sustained stimulus in most cutaneous mechanosensors (Kwan et al., 2009). Slippage of mechanical coupling structures can only partially account for this adaptation, so the balance must be attributed to regulation of the spike generation process that transforms neuronal membrane deformation into action potential (AP) trains (Loewenstein and Mendelson, 1965; Bensmaïa et al., 2005). However, the physiological basis for this modulation has not been identified.

Cytoplasmic $\mathrm{Ca}^{2+}$ controls basic neuronal functions, including membrane excitability and synaptic transmission. In sensory neurons, use-dependent depression of intrinsic membrane excitability is mediated by activity-induced $\left[\mathrm{Ca}^{2+}\right]_{\mathrm{c}}$ elevation and requires the participation of $\mathrm{Ca}^{2+} /$ calmodulin-dependent protein kinase II (CaMKII) (Tang et al., 2012). This ubiquitous $\mathrm{Ca}^{2+} /$ CaM-activated kinase is highly abundant in the nervous system, including peripheral sensory neurons (Carlton, 2002; Kojundzic et al., 2010). Its multimeric structure exposes certain residues (Thr286/287 depending on isoform) to phosphorylation by the activity of adjacent activated subunits. This reaction generates a $\mathrm{Ca}^{2+}$-independent activated state (pCaMKII) that retains enzymatic activity even in the absence of elevated $\mathrm{Ca}^{2+}$, enabling CaMKII to translate neuronal activity into graded levels of sustained kinase activity (Hudmon and Schulman, 2002). Because CaMKII is a multifunctional kinase that has numerous targets in multiple compartments (nucleus, cytoplasm, and membrane) throughout the cell, it can be considered an ideal candidate to function as a master regulator for coordinating neuronal excitability according to past neuronal activity. We therefore tested the hypothesis that sensory neuron CaMKII is the molecular effector for use-dependent plasticity in low threshold mechanosensation that conveys touch. We additionally examined the role of CaMKII in regulating centripetal propagation of AP trains in $\mathrm{A} \beta$-LTMRs and their eventual activation of second-order neurons in the spinal dorsal horn.

\section{Materials and Methods \\ Animals}

Animal experiments were approved by the $\mathrm{Za}$ blocki Veterans Administration Medical Center Animal Studies Subcommittee and Medical College of Wisconsin Institutional Animal Care and Use Committee in accordance with the National Institutes of Health Guidelines for the Care and Use of Laboratory Animals. Animals were housed at $22 \pm 0.5^{\circ} \mathrm{C}$ and $60 \pm 15 \%$ humidity, with a $12 \mathrm{~h}$ light-dark cycle and free access to water. Male Sprague Dawley rats (5-6 weeks old) were purchased from Charles River Laboratories, and male 6- to 24-week-old C57BL/6J mice were purchased from The Jackson Laboratory. Animals in all treatment groups gained weight at a normal rate.

\section{Construction of adeno-associated viral $(A A V)$ vectors}

Plasmids used for recombinant AAV production were obtained from Applied Viromics: (1) self-complementary (sc) AAV expression plasmid ( $\mathrm{pAAV}$-CMV-EGFP) containing a CMVdriven EGFP expression cassette with the simian virus 40 poly(A) signal, flanked by sc AAV2 inverted terminal repeats; (2) pRC8 containing AAV2 replication gene and capsid protein gene from AAV8; and (3) pHelper encoding the adenoviral helper genes. Construction of the plasmid of pEGFP-AIP expressing a fluorescent AIP-based CaMKII inhibitor, which was kindly provided by Dr. Steven H. Green (Department of Biological Sciences, University of Iowa), was achieved by inserting the autocamtide- 2 related inhibitory peptide (AIP; KKALRRQEAVDAL) DNA sequence into the EGFP-C1 vector, which consists of a 22 amino acid linker (SGLRSRAQASNSAVDGTAGPGS) attached to the C terminus of EGFP (Bok et al., 2007). Efficacy of the expressed EGFP-AIP in inhibiting activation of a CRE-reporter construct has previously been demonstrated in cells containing constitutively active CaMKII, and neither CaMKIV nor cAMP-dependent protein kinase was affected (Bok et al., 2007). To construct the scAAV vector containing a chimeric EGFP-AIP expression cassette, we subcloned a DNA fragment containing the AIP sequence from pEGFP-AIP into BsrGI/HpaI sites of pAAV-CMV-EGFP to generate $\mathrm{pAAV}$-CMV-EGFP-AIP that codes the EGFP-AIP fusion protein under the transcriptional control of the CMV promoter. The construct was sequenced to confirm the desired DNA sequence and the correct reading frame. The control vector AAV8-EGFP that contained the linker but lacked the AIP sequence.

\section{$A A V$ vector production}

To permit valid comparison of the effects of the active and control vector, vector quality was tightly regulated to minimize potential secondary effects by impurities and to optimize vector titers. Highly purified stocks of AAV8-EGFP-AIP and AAV8-EGFP vectors were generated by a helper virus-free system, followed by two cycles of $\mathrm{CsCl}_{2}$ gradient purification, according to a previously described protocol (Tang et al., 2012). Final viral preparations were kept in PBS containing $5 \%$ sorbitol (Sigma-Aldrich) at $-80^{\circ} \mathrm{C}$. Encapsidated viral DNA was quantified against plasmid standards by a PicoGreen assay (Invitrogen) following denaturation of the purified AAV particles, and the physical titer was expressed as genome copy number per milliliter (GC/ml) (Tang et al., 2012). Titers of AAV8-EGFP-AIP and AAV8-CMV-EGFP were $9.84 \times 10^{12} \mathrm{GC} / \mathrm{ml}$ and $2.11 \times 10^{13} \mathrm{GC} / \mathrm{ml}$, respectively. The purity of these AAV preparations was confirmed by silver staining (Fig. 1A).

\section{Microinjection of $A A V$ vectors into DRGs}

AAV vector was microinjected into right lumbar L4 and L5 DRGs (Fischer et al., 2011) through the surgically exposed intervertebral foramen that was enlarged by limited removal of laminar bone, using 
Table 1. Antibodies used in immunohistochemistry and Western analysis

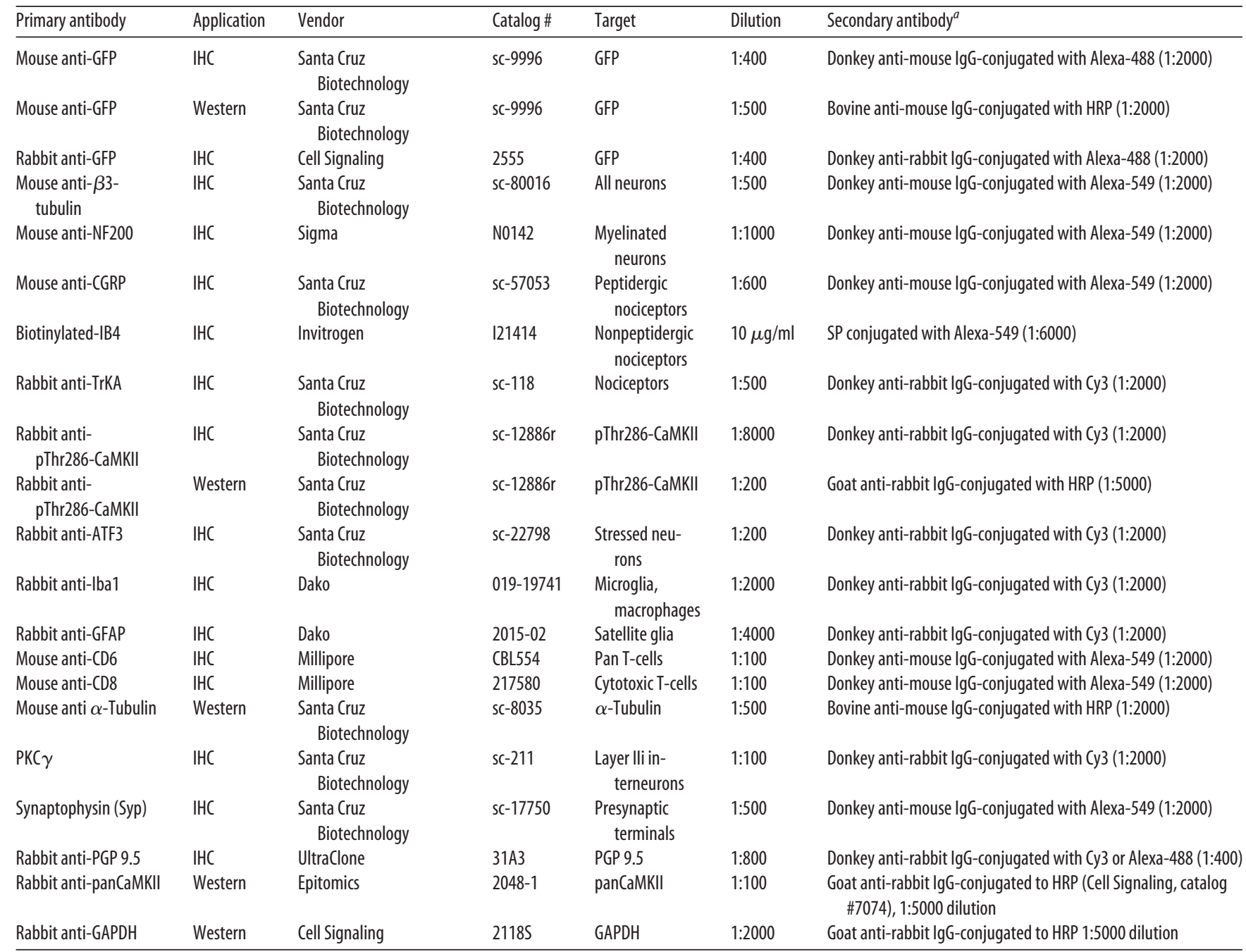

${ }^{a}$ All secondary antibodies are from Jackson ImmunoResearch Laboratories.

a micropipette advanced $50 \mu \mathrm{m}$ into the ganglion. Rats received L4 and L5 DRG injections of either AAV8-EGFP-AIP or AAV8-EGFP (one vector per rat, randomized), consisting of $2 \mu \mathrm{l}$ with adjusted titers containing a total of $2.0 \times 10^{9} \mathrm{GC}$ particles. Piston-driven injection was performed over a 5 min period, after which the wound was closed.

\section{Behavioral examination}

Rats were habituated to the behavioral testing apparatus for $1 \mathrm{~h}$ before data collection. All behavior evaluation was performed by personnel blind to the animals' treatment.

Dynamic mechanical stimulation (brush). Animals were placed on a wire mesh platform, and a camelhair brush with a width of $5 \mathrm{~mm}$ was passed along the bottom of the hindpaw from front to back in a smooth motion at a rate of $\sim 2 \mathrm{~cm} / \mathrm{s}$. Each hindpaw was tested 3 times in alternating fashion. The frequency of withdrawal from the stimulus was recorded.

Cold stimulation. Acetone was applied to the plantar surface 3 times in alternating fashion and the frequency of withdrawal determined.

Mild mechanical stimulation (von Frey). Withdrawal threshold was determined using calibrated monofilaments with forces of $0.3,0.5,0.8,1.0,2.8$, $5,9,14$, and $24 \mathrm{~g}$, applied in an up-down fashion, allowing calculation of the $50 \%$ withdrawal threshold (Chaplan et al., 1994). Rats not responding to any filament were assigned a score of $25 \mathrm{~g}$.

Heat stimulation. The time elapsed to withdraw from a radiant heat source was measured 4 times, and the withdrawal latency values were averaged.
Noxious mechanical stimulation (pin). A $22 \mathrm{~g}$ spinal anesthesia needle was applied 5 times to the plantar surface with enough force to indent but not puncture the skin and repeated after at least $2 \mathrm{~min}$. This evoked either a simple withdrawal response with immediate return of the foot to the cage floor, or a response characterized by sustained elevation with grooming and possibly shaking, lasting at least $1 \mathrm{~s}$. This hyperalgesic response has been associated specifically with an aversive experience (Wu et al., 2010).

Motor testing (rotarod). After initial training sessions, rats were placed on a drum and allowed to acclimate before gradually accelerating rotation from 4 to $40 \mathrm{rpm}$ over $300 \mathrm{~s}$, and the time elapsed before falling was recorded.

Analysis. Time-series data within each group were analyzed for an effect of time by repeated-measures ANOVA and post hoc comparisons between baseline (i.e., day 0) using Dunnett's test for continuously variable measures (von Frey, heat, and rotorad), or by nonparametric Friedman's ANOVA with post hoc comparisons to baseline using Dunn's test for discrete variable measures (cold and pin). Differences between groups using the area under the curve (AUC) for the behavioral score normalized against the baseline day. AUC scores for each group were compared using a $t$ test or the MannWhitney $U$ test.

\section{Spinal nerve ligation (SNL) model}

During isoflurane anesthesia, rat L5 and L6 spinal nerves were exposed unilaterally in the paravertebral space, ligated, and transected, followed by wound closure. Control animals had anesthesia and skin incision alone. 
A

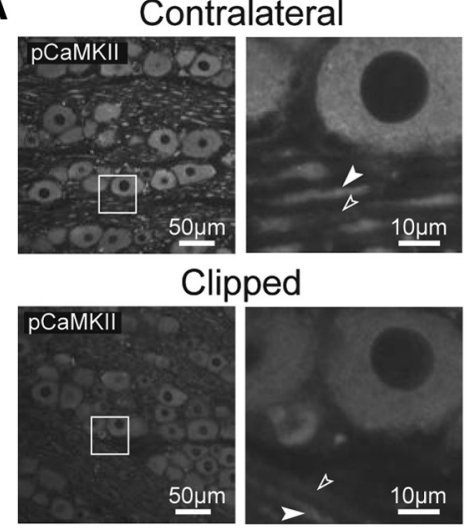

C

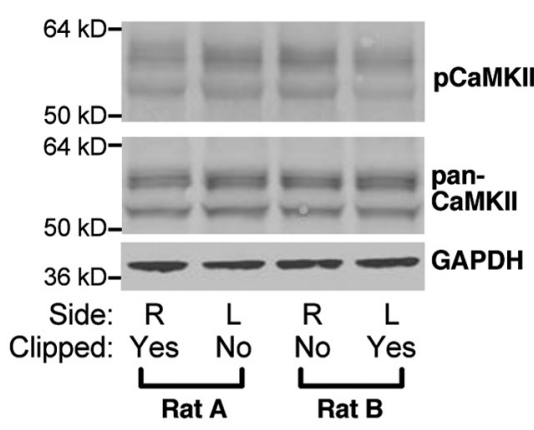

$\mathbf{F}$

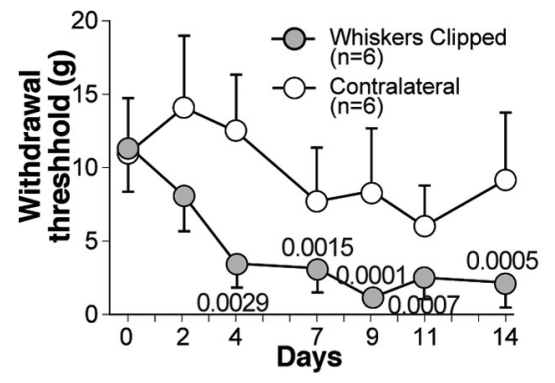

H

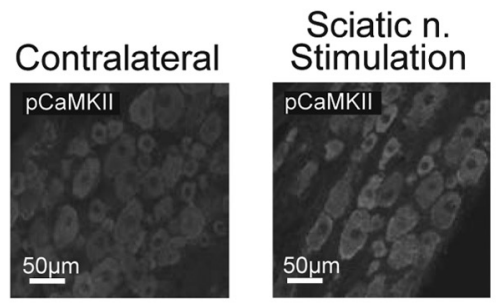

D
B
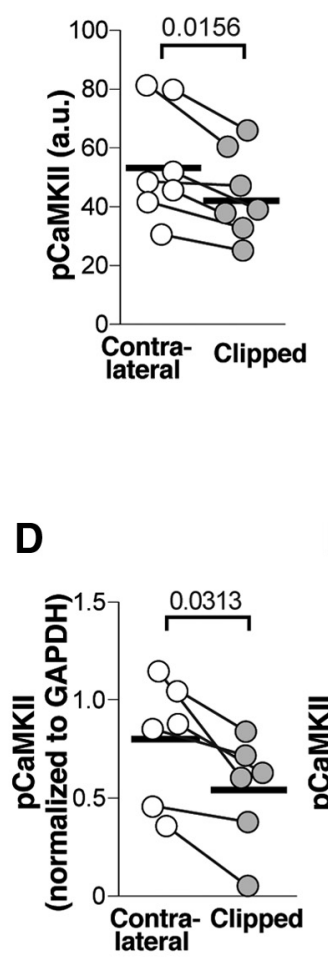

E

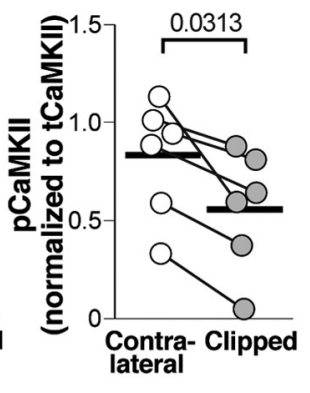

G
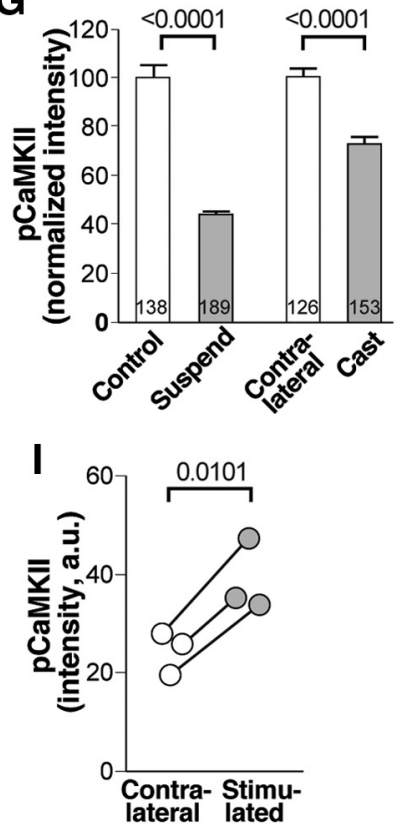

Sensory deprivation models

During brief isoflurane anesthesia, whiskers were removed unilaterally by electrical shaver while avoiding direct contact with the skin every $2-3 \mathrm{~d}$ throughout the 2 week treatment period. The side (right vs left) was randomized. Before shaving, threshold for withdrawal from von Frey mechanical stimulation in the mystacial pads bilaterally was tested as described above.

For tail suspension (Morey-Holton and Globus, 2002), the tail was tethered to a swivel that elevates the hindpaws off the cage floor for $14 \mathrm{~d}$. Brush testing was performed in control animals just after placing them in tail suspension.

For cast immobilization (Guo et al., 2004), one hindlimb was placed in a synthetic resin cast lined with cotton that enclosed the metatarsals at its inferior-most extent and joined a circumferential component at the level of the abdomen, retaining the limb's normal standing posture, and maintained for $10 \mathrm{~d}$.

As a positive control, the sciatic nerve was exposed at mid-thigh level during isoflurane anesthesia and stimulated (1 ms pulse duration, $20 \mathrm{~Hz}, 10 \mathrm{~min}$ ) via platinum electrodes at twice the threshold for movement.

\section{Immunohistochemistry}

L4/L5 DRGs, corresponding spinal cord, sciatic nerve, and ipsilateral plantar skin were removed and fixed in $4 \%$ PFA made in $0.1 \mathrm{M} \mathrm{PBS}\left(4^{\circ} \mathrm{C}, \mathrm{pH}\right.$ 7.4 ), embedded with paraffin, sectioned ( $5 \mu \mathrm{m})$, and immunostained sequentially with primary antibodies followed by the specified Alexa-488or 549-conjugated secondary antibodies (Table 1). For each experiment, tissues from compared groups were prepared and stained concurrently, and image capture parameters were unchanged throughout the analysis. For harvest of tissue in which pCaMKII levels would be determined or skin histology would be examined, cardiac perfusion was initiated during isoflurane anesthesia using oxygenated $\mathrm{Ca}^{2+}$-free solution before perfusion by PFA, followed by $4 \mathrm{~h}$ after fixing in the same solution at $4^{\circ} \mathrm{C}$, before storage in $\operatorname{PBS}\left(4^{\circ} \mathrm{C}\right)$.

Cytoplasmic immunofluorescence was measured, in a blinded fashion, individually for all neurons in a field with a nuclear profile. Neurons were considered to be transduced if EGFP immunofluorescence exceeded a background standard from naive neurons by $>2$ SDs. For identifying pCaMKII, a phospho-antibody (Santa Cruz Biotechnology, \#12886r) was used as a reporter of CaMKII Thr286/287 autophosphorylation. We confirmed that this phosphoantibody recognizes all four CaMKII isoforms by Western blot against purified single-

Figure 2. Sensory deprivation increases cutaneous sensitivity and decreases pCaMKII levels. A, Whisker clipping (14 d) reduces pCaMKII levels in ipsilateral trigeminal ganglia sensory neuron somata and axons. Areas enclosed in the white square are enlarged (right panels), which show that increased pCaMKIl staining is present in cytoplasm of axons (filled arrowheads) and neuronal somata, but background staining in axonal myelin (open arrowheads) and interstitial areas is similar. $\boldsymbol{B}$, Data for individual rats $(n=7)$ show reduced pCaMKII levels (medians of $n=26-52$ neurons; paired $t$ test) on whisker-deprived side using immunofluorescence. Error bars indicate group means. C, Western blots for individual trigeminal ganglia isolated from 2 rats also indicate reduced pCaMKII levels with whisker deprivation (paired $t$ tests). Group data show decreased Thr286/287 phosphoimmunostaining normalized to GAPDH (total protein, $\boldsymbol{D}$ ) and to total CaMKII (immunostained for pan-CaMKII, E). Error bars indicate group means. $\boldsymbol{F}$, Whisker removal decreased the withdrawal threshold from mechanical stimulation (repeated-measures ANOVA, $p=0.0001 ; p$ values are shown for significant paired comparisons to day 0 ), without effect on the unshaved side ( $p=$ 0.3627). Data are mean \pm SEM. G, Tail suspension (2 rats) depressed average pCaMKII levels in sensory neuron somata normalized

\section{$\leftarrow$}

against the average of levels in a control animal housed normally. Cast enclosure of the foot and leg (1 rat) depressed average $\mathrm{pCaMKII}$ normalized against the average in contralateral DRGs. $n$ for neurons shown in bar. Data are mean \pm SEM. $\boldsymbol{H}$, Sciatic nerve stimulation (10 min, $20 \mathrm{~Hz}$ ) during inhalation anesthesia elevated pCaMKII levels in ipsilateral DRG neurons. I, Data for individual rats show increased pCaMKII fluorescence intensity following sciatic nerve stimulation. 
A

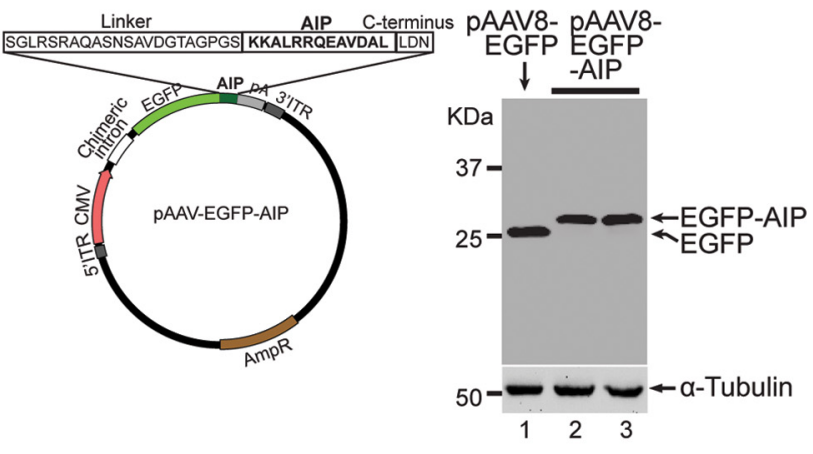

C Dorsal Root Ganglion
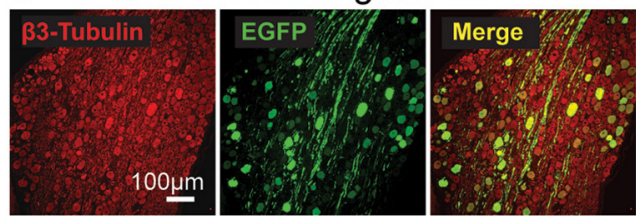

D Sciatic Nerve
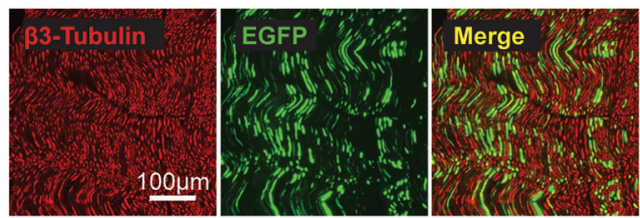

Figure 3. AAV virus encoding EGFP-AIP produces efficient transduction of sensory neurons in vivo. $A$, Recombinant scAAV plasmid encoding EGFP-AIP with linker-AIP-C terminus amino acid sequence. $\boldsymbol{B}$, The stability of the peptide fusion in AAV-transduced HEK293T cells is shown by the expected MW shift for EGFP-AIP fusion (duplicate cultures in lanes 2 and 3) versus EGFP expression alone (lane 1); $\alpha$-tubulin loading control (bottom). C, Dorsal root ganglion section shows successful transduction of neuronal somata and axons (costained for $\beta 3$-tubulin) by AAV8-EGFP-AIP. $D$, Sciatic nerve section after DRG injection of AAV8-EFP-AIP shows EGFP-AIP expression in $\beta 3$-tubulin-positive fibers.

isoform recombinant CaMKII proteins. Additionally, we showed it to be selective for only proteins that had been autophosphorylated in vitro when $\mathrm{CaCl}_{2}(1 \mathrm{mM})$, calmodulin $(5 \mu \mathrm{M})$, and adenosine triphosphate $(100 \mu \mathrm{M})$ were present in the buffer solution (in mM as follows: HEPES $50, \mathrm{NaCl} 100$, DTT 2, $\mathrm{MgCl}_{2} 10, \mathrm{CaCl}_{2} 1, \mathrm{pH}$ 7.4) (Fig. 1B). To determine the intensity of pCaMKII immunostaining, cytoplasmic fluorescence intensity was normalized against the average of the relevant control (i.e., neurons contralateral to whisker clipping or hindlimb casting, or a control littermate for tail-suspended rat). For whisker clipping, data were derived from 2 fields per subject and a median of 30 neurons per field. For tail suspension, pCaMKII data were derived from 2 rats, L4 and L5 DRGs, 24 fields, and a median of 6 neurons per field, normalized against the average of levels in a control animal housed normally ( 21 fields, 6 neurons per field). For cast enclosure of the foot and leg, pCaMKII levels in the L5 DRG of a single rat ( 17 fields, 8 neurons per field) were normalized against the average in contralateral DRGs ( 15 fields, median of 10 neurons per field). For sciatic stimulation, pCaMKII data were derived from 2-6 fields bilaterally in each of 3 subjects, and a median of 7 neurons per field.

To test the effect of EGFP or EGFP-AIP expression on pCaMKII fluorescence levels, cytoplasmic pCaMKII immunofluorescence intensity of transduced neurons was normalized against the average fluorescence of nontransduced neurons in the same field. Neuronal size influences pCaMKII fluorescence levels (data not shown), so only medium/large neurons (area $>700 \mu \mathrm{m}^{2}$ of neuronal profiles that included the nucleus) were evaluated. Data were derived from L4 and L5 DRGs of 2 animals, 4-7 fields per vector, 4-12 neurons per field.

For determining transduction efficiency (i.e., the percentage of EGFPexpressing neurons of all neurons identified with $\beta 3$-tubulin immunofluorescence), at least three nonconsecutive ipsilateral sections were imaged per DRG, and six DRGs were examined per vector. Somatic size was determined from the area of neuronal somata.

For the evaluation of cutaneous innervation, hindpaws were removed at the ankles following death and transcardial perfusion. Removed skin was infiltrated with $30 \%$ sucrose in PBS and frozen-sectioned by cryostat $(14 \mu \mathrm{m})$ perpendicular to the epidermal surfaces. Multiple slides with consecutive sections were processed for double labeling. All sections were analyzed and digital images captured using a confocal microscope equipped with conventional fluorescence filters and a high-speed camera. Each section was examined, with particular attention to identifying terminals and fibers typical of various sensory neuron types.

\section{Western blotting}

To avoid influencing CaMKII activation, trigeminal ganglia were harvested during cardiac perfusion using oxygenated, cold, $\mathrm{Ca}^{2+}$-free buffer, during which ganglia were excised and then frozen in liquid $\mathrm{N}_{2}$. Harvested ganglia were mechanically disrupted with a glass-on-glass ho- mogenizer using $150 \mu \mathrm{l}$ of modified RIPA buffer (50 mu Tris, $\mathrm{pH}$ 8.0, 150 mм NaCl, $1 \%$ NP- $40,0.5 \%$ sodium deoxycholate, $0.1 \%$ SDS, 1 mм sodium orthovanadate, $1 \mathrm{~mm}$ sodium fluoride, $2 \mathrm{~mm}$ EDTA, 1 mM EGTA) containing $1 \times$ protease inhibitors (Calbiochem, catalog \#539137) plus $1 \times$ phosphatase inhibitors (Calbiochem, catalog \#524624). The RIPA buffer was confirmed not to inhibit CaMKII enzymatic activity when diluted using an in vitro kinase assay. After incubation on ice, samples were centrifuged at $1000 \times g$ for $5 \mathrm{~min}$ at $4^{\circ} \mathrm{C}$ to remove cell debris and stored at $-80^{\circ} \mathrm{C}$. Total protein concentration of the supernatants was measured by Pierce bicinchoninic acid protein assay kit using BSA as a standard (Thermo Scientific, 23227). Protein supernatants with equal amounts of protein $(30 \mu \mathrm{g})$ were separated using SDS-PAGE and transferred onto nitrocellulose membrane. After blocking with $10 \%$ milk in TBST (Tris-buffered saline $+0.1 \%$ Tween 20 ), blots were sequentially probed with primary antibodies and corresponding secondary antibodies (Table 1). Western Lighting Plus-ECC (PerkinElmer, \#NEL104001 EA) or Odyssey Infrared Imager (Li-Cor) was used for the detection of the protein bands. Intensity of protein bands was assessed using Image Studio version 2.1 (Li-Cor). Phospo-CaMKII amount was normalized based on the signal intensity of either pan-CaMKII or GAPDH.

\section{Neuronal dissociation and plating}

As described previously (Tang et al., 2012), L4 and L5 DRGs were harvested during isoflurane anesthesia and incubated in $0.1 \mathrm{mg} / \mathrm{ml}$ collagenase followed by $0.25 \%$ trypsin and $0.125 \%$ DNase in DMEM. Isolated neurons were cultured in medium containing $0.5 \mathrm{~mm}$ glutamine, 10 $\mathrm{ng} / \mathrm{ml}$ nerve growth factor $7 \mathrm{~S}$, and $0.02 \mathrm{mg} / \mathrm{ml}$ gentamicin, maintained at $37^{\circ} \mathrm{C}$ in $5 \% \mathrm{CO}_{2}$ in air for $2 \mathrm{~h}$, and studied no later than $6 \mathrm{~h}$ after harvest.

\section{Measurement of cytoplasmic $\mathrm{Ca}^{2+}$ concentration}

Dissociated neurons were loaded with fura-2 AM ( $5 \mu \mathrm{M})$ and maintained in Tyrode's solution containing the following (in $\mathrm{mM}$ ): $\mathrm{NaCl} 140, \mathrm{KCl} 4$, $\mathrm{CaCl}_{2}$ 2, glucose $10, \mathrm{MgCl}_{2} 2$, and HEPES 10, with an osmolarity of 297-300 mOsm, pH 7.4. Transduced neurons were identified by EGFP fluorescence. The fluorophore was excited alternately with $340 \mathrm{~nm}$ and $380 \mathrm{~nm}$ wavelength illumination, and images were acquired at $510 \mathrm{~nm}$ using a cooled 12-bit digital at a rate of $3 \mathrm{~Hz}$. The fluorescence ratio $\mathrm{R}$ for individual neurons was determined as the intensity of emission during $340 \mathrm{~nm}$ excitation $\left(\mathrm{I}_{340}\right)$ divided by $\mathrm{I}_{380}$, and the $\mathrm{Ca}^{2+}$ concentration was estimated by as $\left(\mathrm{K}_{\mathrm{d}}\right)(\beta)\left(\mathrm{R}-\mathrm{R}_{\min }\right) /\left(\mathrm{R}_{\max }-\mathrm{R}\right)$, where $\beta$ is the ratio of $\mathrm{I}_{380}$ at zero and saturating $\mathrm{Ca}^{2+}$ concentrations. Values of $\mathrm{R}_{\min }, \mathrm{R}_{\max }, \beta$, and $\mathrm{K}_{\mathrm{d}}$ were $0.38,8.49$, and 9.54, and $224 \mathrm{~nm}$ (Grynkiewicz et al., 1985). Activation of voltage-gated $\mathrm{Ca}^{2+}$ channels was triggered by $\mathrm{K}^{+}(35 \mathrm{~mm}$, $1 \mathrm{~s}$ ), which limits involvement of mitochondrial $\mathrm{Ca}^{2+}$ buffering (Gemes et al., 2012), and plasma membrane $\mathrm{Ca}^{2+}$-ATPase influence was eliminated by applying Tyrode's with $\mathrm{pH} 8.8$ during depolarization (Gemes et al., 2012) while stable intracellular $\left[\mathrm{Ca}^{2+}\right]_{\mathrm{C}}$ was maintained by simulta- 

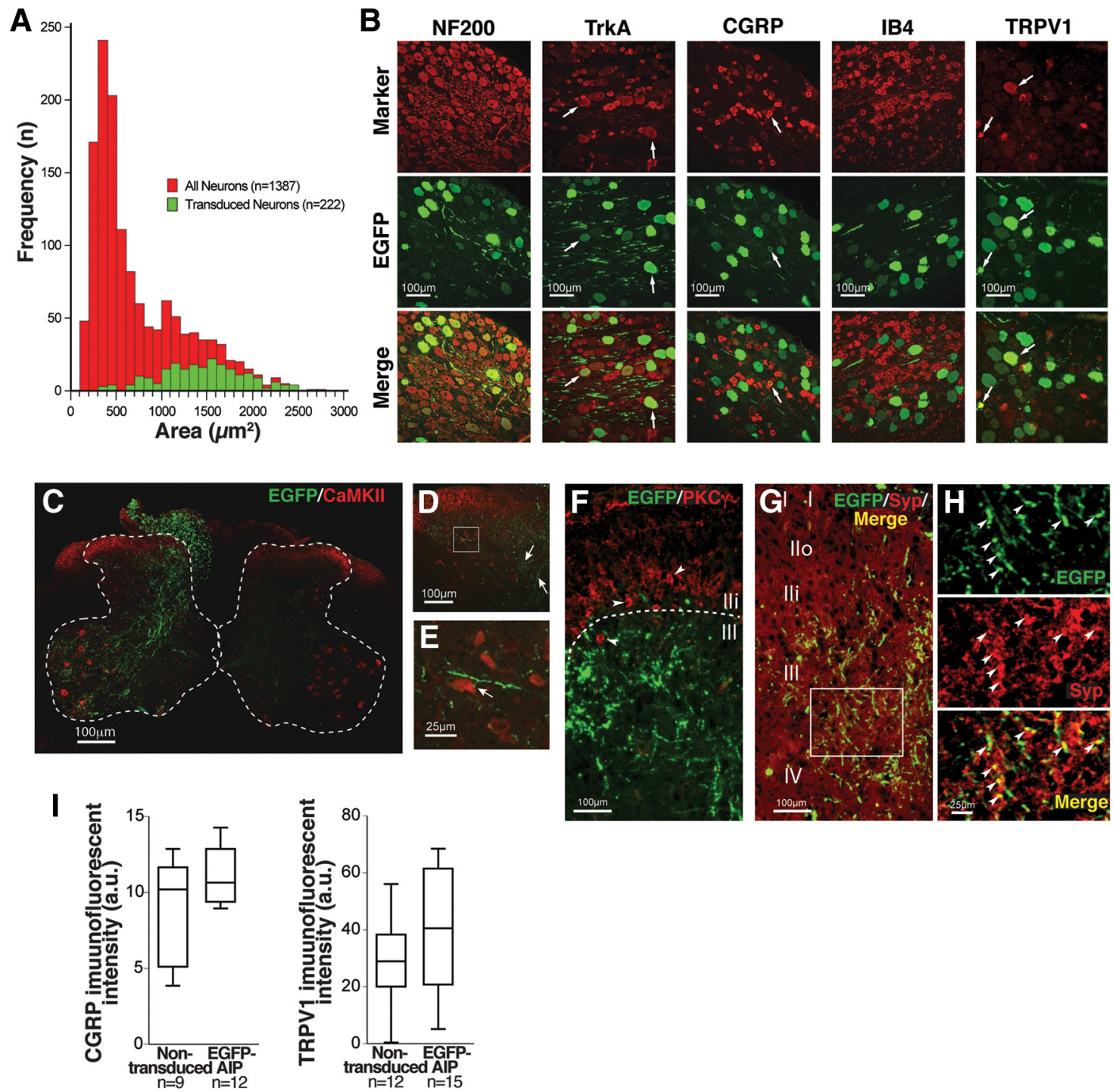

Figure 4. Characterization of DRG neurons transduced by AAV8-EGFP-AIP. A, Size distribution of neuronal somata transduced by AAV8-EGFP-AIP (4 weeks after injection) is shifted toward larger areas compared with the total population identified by $\beta 3$-tubulin staining. $\boldsymbol{B}$, Representative DRG fields show immunostaining with markers NF200, TrkA, (GRP, IB4, and TRPV1 (top panels), EGFP-AIP immunostaining (middle panels), and merged images (bottom panels). Transduced neurons all show NF200 staining typical of large/myelinated neurons. Some are weakly positive for TrkA (arrows) typical of nociceptive neurons. Few transduced neurons show CGRP (arrow) or IB4 immunoreactivity typical of peptidergic and nonpeptidergic nociceptors, or TRPV1 (arrows). C, Transverse spinal cord section at L4/L5, 4 weeks following intraganglionic AAV8-EGFP-AIP, shows transduced fibers (EGFP-AIP immunofluorescence) distributing to deeper dorsal horn laminae. Counterstaining for pCaMKII identifies superficial lamina (I,II), and dorsal and ventral horn neurons. Spinal cord gray matter is outlined. $\boldsymbol{D}$, At higher magnification, the dorsal horn ipsilateral to vector injection reveals EGFP-AIP expression in synaptic varicosities (arrows). No transduced fibers extend into the superficial dorsal horn. $\boldsymbol{E}$, The boxed area in $\boldsymbol{D}$ shown at still higher magnification illustrates a transduced sensory neuron process (arrow) synapsing with a dorsal horn neuron. All dorsal horn neurons lack EGFP expression. $\boldsymbol{F}$, Transduced fibers extend up to the area stained by inner lamina II (lli) marker PKC $\gamma$. G, EGFP is colocalized with the presynaptic marker synaptophysin (Syp). $\boldsymbol{H}$, The boxed area in panel $\mathbf{G}$ is shown in higher magnification, with arrowheads showing colocalization. $\boldsymbol{I}$, CGRP and TRPV1 immunofluorescence in the DRG were not affected by EGFP-AIP expression in medium/large neurons ( $>700 \mu \mathrm{m}^{2}$ ) compared with nontransduced neurons. $p=0.39$, for CGRP (MannWhitney). $p=0.164$, for TRPV1 (Mann-Whitney). Data are from the DRGs shown in $\boldsymbol{B}$.

neously reducing bath $\mathrm{Ca}^{2+}$ concentration to $0.25 \mathrm{~mm}$ (Duncan et al., 2013).

\section{Whole-cell recording of dissociated neurons}

Neurons were studied using the whole-cell configuration of the patchclamp technique at room temperature as described previously (Tang et al., 2012). Tyrode's solution was used for external bath solution. The internal pipette solution contained the following (in $\mathrm{mM}$ ): potassium gluconate $135, \mathrm{KCl} 5, \mathrm{MgCl}_{2}$ 2, EGTA 0.2 , HEPES 10, $\mathrm{Na}_{2}$ phosphocreatine $10, \mathrm{Mg}$-ATP $4, \mathrm{Na}_{2}$-GTP 0.3 , at $\mathrm{pH}$ of 7.2 with $\mathrm{KOH}$ and osmolarity of 296-300 mOsm. Whole-cell recordings were made with patch pipettes $(2-4 \mathrm{M} \Omega)$; signals were filtered at $2 \mathrm{kHz}$ and sampled at $10 \mathrm{kHz}$. The rheobase current necessary for generating an AP was determined for each neuron by injecting a series of depolarizing cur- rents (200 ms) with progressively greater amplitudes (20 pA increments). To measure neuronal excitability, a current of $1.5 \times$ rheobase was injected for $1 \mathrm{~s}$ and the number of APs was tabulated. To identify the influence of a conditioning depolarization, the neuron was depolarized to $0 \mathrm{mV}$ for $2 \mathrm{~s}$ during voltage clamp, and $15 \mathrm{~s}$ later the number of APs generated during $1.5 \times$ rheobase current injection was again tested.

\section{Sharp electrode intracellular recording}

Intact ganglia with dorsal roots attached were harvested by laminectomy during cardiac perfusion using ice-cold Tyrode's solution and placed in aCSF (in mM as follows): $\mathrm{NaCl} 128, \mathrm{KCl} 3.5, \mathrm{MgCl}_{2} 1.2, \mathrm{CaCl}_{2} 2.3$, $\mathrm{NaH}_{2} \mathrm{PO}_{4} 1.2, \mathrm{NaHCO}_{3} 24$, glucose 11 , aerated with $5 \% \mathrm{CO}_{2}$ in $\mathrm{O}_{2}, \mathrm{pH}$ 7.35 , at $35^{\circ} \mathrm{C}$. Microelectrodes $(60-90 \mathrm{M} \Omega)$ were filled with $2 \mathrm{M} \mathrm{K}^{+}$ 
A Skin: Merkel Endings
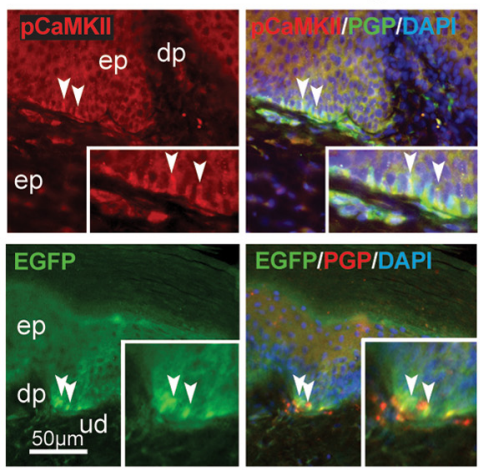

D DRG: AAV8-EGFP-AIP
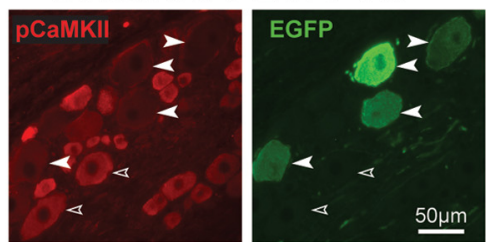

DRG: AAV8-EGFP
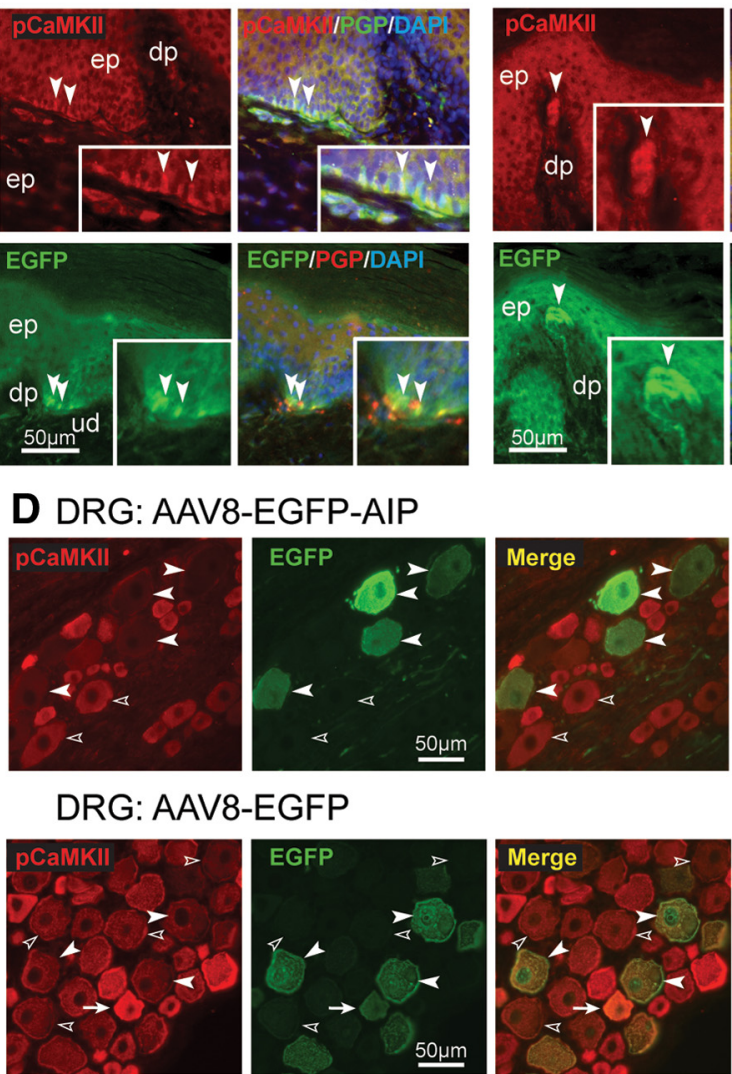

B Skin: Meissner Corpuscles
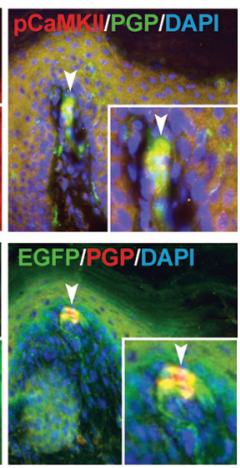

C Skin: Lanceolate Endings
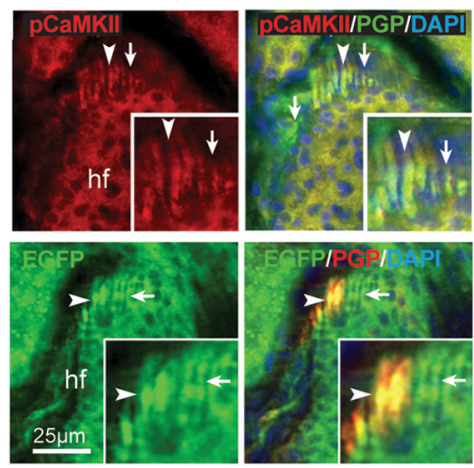

E

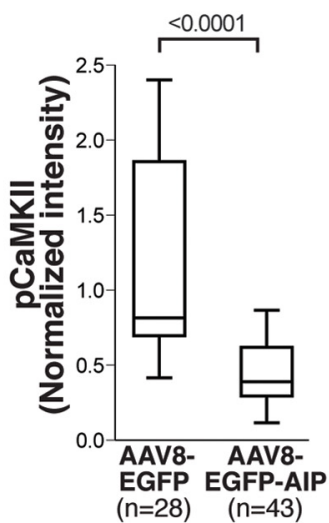

Figure 5. Transduced sensory neurons terminate in A $\beta$-LTMR endings. Tissue was harvested following an ipsilateral injection of AAV8-EGFP-AIP into L4 and L5 DRGs. A, Merkel endings (arrowheads) are identified at the base of the epidermis (ep) in glabrous plantar skin. Top left, pCaMKII immunofluorescence. Top right, Coexpression with PGP and DAPI. Bottom left, EGFP immunostaining in a different field. Bottom right, Coexpression with PGP and DAPI. ud, Upper dermis; dp, dermal papilla. B, Meissner corpuscles (arrowheads) in the dermal papillae were also immunostained for pCaMKII and EGFP-AIP expression in glabrous skin. $\boldsymbol{C}$, In hindpaw dorsal hairy skin, large and small lanceolate endings (arrowheads and arrows) in palisades around hair follicle (hf) show pCaMKII and EGFP expression. D, Transduction by the AAV8-EGFP-AIP vector (filled arrowheads) reduced pCaMKII levels compared with nontransduced neurons (open arrowheads, top), but this was not seen in neurons transduced by the control AAV8-EGFP vector (bottom). Although an occasional small/medium neuron is transduced (arrow), most transduced neurons were large. $\boldsymbol{E}$, Quantification shows that pCaMKII fluorescence level in transduced neurons (from 4 injected DRGs), normalized against average pCaMKII fluorescence in neighboring nontransduced neurons, is depressed by EGFP-AIP expression (Wilcoxon Signed Rank, $p<0.0001$ ), but not by EGFP expression $(p=0.32$ ), resulting in a difference in normalized fluorescence (Mann-Whitney, $p<0.0001$ ).

acetate buffered with 10 mM HEPES, pH 7.2. Recorded neurons had a diameter of $>30 \mu \mathrm{m}$ and resting $\mathrm{V}_{\mathrm{M}}<-45 \mathrm{mV}$. Somatic APs were generated either by current injection through the recording electrode or by dorsal root axonal stimulation. Although this is antidromic to sensory traffic, the influence of the sensory neuron T-junction on propagation of AP trains does not depend on direction of passage (Lüscher et al., 1994). Input resistance $\left(\mathrm{R}_{\mathrm{in}}\right)$ was determined by injection of hyperpolarizing current $(0.5 \mathrm{nA}, 10 \mathrm{~ms})$ at $15 \mathrm{~ms}$ after the last axonal stimulus and every $50 \mathrm{~ms}$ thereafter, following trains at the fastest frequency at which each cell could fire 20 full somatic APs. Agents were applied either by $30 \mathrm{~min}$ incubation (myristoyl or myristoylated AIP [mAIP]) or bath change (DMSO, and KN-92 or KN-93, Millipore). Other recordings using the same sharp electrode technique were made from neurons $(>30 \mu \mathrm{m}$ diameter) following DRG dissociation as described above.

\section{Skin-nerve single-unit recording}

The sural nerve and hairy and glabrous skin of the fourth and fifth digits were removed from anesthetized male 6- to 24-week-old C57BL/6J mice, pinned down in a bath containing extracellular buffer composed of (in mM) the following: $\mathrm{NaCl} 123, \mathrm{KCl} 3.5, \mathrm{MgSO}_{4} 0.7, \mathrm{NaH}_{2} \mathrm{PO}_{4} 1.7, \mathrm{CaCl}_{2}$ 2.0, Na gluconate 9.5, glucose 5.5, sucrose 7.5, and HEPES 10, pH 7.45, osmolality $290 \mathrm{mOsm}$, aerated with $\mathrm{O}_{2}$ and maintained at $32^{\circ} \mathrm{C}$, and containing either $\mathrm{KN}-92$ or $\mathrm{KN}-93$ (both $10 \mu \mathrm{M}$, each with $0.125 \%$ DMSO) in a blinded fashion. Teased filaments were placed on an $\mathrm{AgCl}$ electrode, and the skin preparation was probed mechanically with a blunt glass rod to identify individual mechanically sensitive units. Units in- cluded for study had $\mathrm{A} \beta$ conduction velocity $(>10 \mathrm{~m} / \mathrm{s})$. Units that fired throughout mechanical stimulation were classified as slowly adapting, whereas those that ceased firing during the onset of the stimulus were classified as rapidly adapting. The most sensitive part of the receptive field was subjected to a $75 \mathrm{mN}$ force $(10 \mathrm{~s})$ applied via a feedbackcontrolled stimulator with a $1 \mathrm{~mm}$ probe. This force was chosen because it is in the midrange of the force/activity relationship for $\mathrm{A} \beta$-LTMRs (Garrison et al., 2012). To measure firing rate adaptation, the interspike interval (ISI) was converted to instantaneous frequency (1/ISI), which was plotted against time and fitted with a simple one-phase exponential decay to provide a time constant $(\tau)$ that was used to characterize adaptation. Treatment groups did not differ in $\mathrm{CV}$, mechanical force firing threshold (determined by von Frey fibers), or signal-to-noise ratio.

\section{Dorsal horn slice recording}

Anesthetized adult rats were perfused with ice-cold sucrose-substituted aCSF saturated with $20: 1 \mathrm{O}_{2}: \mathrm{CO}_{2}$, the lumbar cord was removed, and transverse (400- to $500-\mu \mathrm{m}$-thick) slices with attached dorsal roots are prepared by vibratome and incubated in standard aCSF. Slices were superfused with aCSF (room temperature). Neurons were recorded in the dorsal horn lamina I, recognized as a translucent bands under brightfield imaging, and chosen as projection neurons characterized by location, morphology, and back-labeling from parabrachial nucleus injection. Whole-cell recordings were obtained with borosilicate pipettes $(3-5 \mathrm{M} \Omega$ ) under infrared DIC microscopy. Signals were filtered at $2 \mathrm{kHz}$ and sampled at $10 \mathrm{kHz}$. For recording miniature EPSCs (mEPSCs), the external 
A von Frey

B Pin
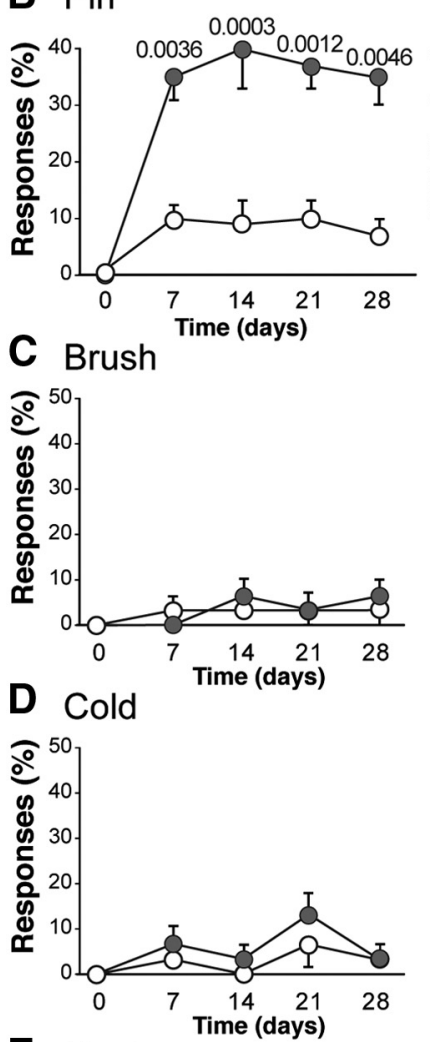

E Heat

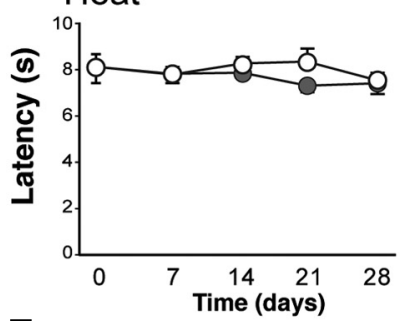

F Rotarod

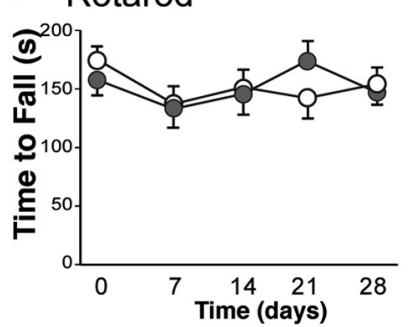

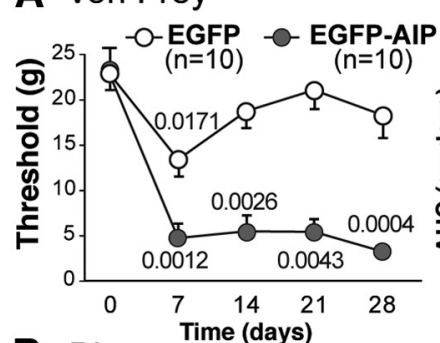
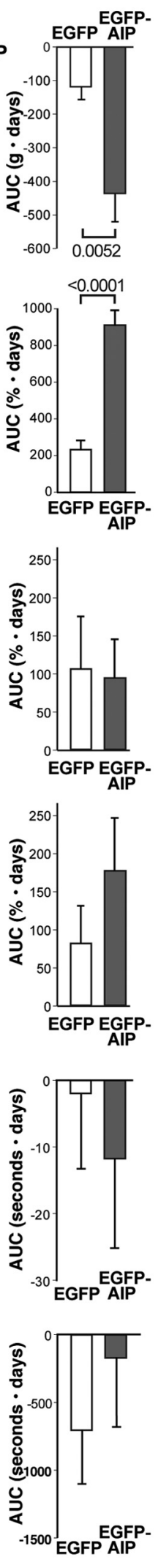

Figure 6. Behavioral effects of transduction of $L 4$ and $L 5 D R G A B$-LTMRs. A, Plantar sensory testing of threshold for withdrawal from mild touch (von Frey fibers) showed only a temporary decrease ( $p=0.0219)$ after AAV8-EGFP injection, whereas AAV8-EGFP-AIP caused a persistent decrease $(p<0.0001)$, and AUC (normalized to day 0 ) confirmed differences between the vectors. $\boldsymbol{B}$, The frequency of hyperalgesia behavior after intense mechanical stimulation with a pin increased after AAV8-EGFP-AIP ( $p=0.0002)$ but not after AAV8-EGFP ( $p=0.1219)$, with solution consisted of ACSF supplemented with picrotoxin and strychnine. TTX had no effect on nonevoked EPSC frequency $(1.09 \pm 0.41 \mathrm{~Hz}$ baseline vs $1.03 \pm 0.27 \mathrm{~Hz}$ with TTX, $p=0.87)$ or amplitude $(14.7 \pm 2.2$ pA baseline vs $15.4 \pm 1.5 \mathrm{pA}$ with TTX, $p=0.85)$, so bath TTX was not needed for recording mEPSCs. Evoked currents were generated by stimulating with contact bipolar electrode ( $1 \mathrm{~mm}$ span) with low current (40-200 nA), anatomically isolating A $\beta$-LTMR afferents at the medial border of lamina III. Evoked currents were sensitive to AMPA blocker CNQX $(20 \mu \mathrm{M})$ and NMDA blocker AP-5 (50 $\mu \mathrm{M}, n=3)$.

\section{Data analysis}

Group sizes were initially estimated on the basis of prior experience with the techniques. No samples or animals were excluded from analysis, except where stated (e.g., in restricted neuronal size groups). Assignment of animals and cells to treatments was randomized. Data are reported and displayed as mean \pm SEM unless otherwise stated. Size distributions of neurons transduced by AAV8-EGFP-AIP versus the total population were compared by the Kolmogorov-Smirnov test. Groups were compared by either $t$ test or ANOVA with post hoc comparisons by Newmann-Keuls test. Nonparametric analysis was performed when data were not normally distributed, for which Tukey boxplots show median and first and third quartiles, with whiskers denoting the lowest datum still within $1.5 \times$ the interquartile range of the first quartile and the highest datum still within $1.5 \times$ the interquartile range of the third quartile. The number of APs propagated at different stimulation rates was compared between mAIP application and control by linear regression. Behavioral analysis is described above.

\section{Results}

\section{CaMKII encodes sensory neuron activity}

We first tested the role of CaMKII as a neuronal activity sensor by measuring levels of the activated form (pCaMKII) in primary sensory neurons that had been deprived of natural mechanical stimulation. Clipping whiskers reduces activation of neurons with somata in the maxillary lobe of the trigeminal ganglion and is an established model for studying the role of neuronal activity in mechanosensation. Whisker clipping resulted in reduced pCaMKII unilaterally on the clipped side determined by immunofluorescence (Fig. 2A,B) and Western blotting (Fig. 2C-E), which was accompanied by increased sensitivity to cutaneous mechanical stimuli (Fig. $2 F$ ). The tail suspension model prevents mechanical stimulation of the plantar surface of the hindpaws (Morey-Holton and Globus, 2002). After 2 weeks, this increased mechanosensitivity (probability of paw withdrawal from innocuous touch with a brush $60 \pm 10 \%, n=5$, vs $4 \pm 4 \%$ in controls, $n=4 ; p=0.002$ ). Lowering the animals into contact with the cage floor caused incessant agitated ambulation and foot grooming that prevented further behavioral testing. Neurons from the L4 DRGs innervating the plantar skin showed diminished pCaMKII after tail suspension (Fig. 2G). Similar changes were seen in the hindlimb cast model (Fig. $2 G$ ), which prevents sensory activation other than contact with the padded interior of the cast, and also results in mechanical hypersensitivity (Guo et al., 2004). In contrast, increased neuronal activation by electrically stimulating the sciatic nerve during inhalation anesthesia elevated sensory neuron pCaMKII (Fig. $2 \mathrm{H}, \mathrm{I}$ ). These findings establish the sensitivity of autophosphorylated CaMKII levels to

group differences confirmed by AUC. $p$ values are shown for significant paired comparisons to day 0 . There were no changes in responses to dynamic mechanical stimulation $(\boldsymbol{C})$, cold $(\boldsymbol{D})$, or heat $(\boldsymbol{E})$, or in motor ability $(\boldsymbol{F})$ after either vector (dynamic mechanical: AAV8-EGFP, $p=0.8874$; AAV8-EGFP-AIP, $p=0.1546$; cold: AAV8-EGFP, $p=0.4060$; AAV8-EGFP-AIP, $p=$ 0.1046; heat: AAV8-EGFP, $p=0.4988$; AAV8-EGFP-AIP, $p=0.5289$; rotarod: AAV8-EGFP, $p=$ 0.2665 ; AAV8-EGFP-AIP, $p=0.2034$ ). AUC across the 4 week testing period for measures normalized to day 0 showed no differences between for these measures. Data are mean \pm SEM. 
preceding natural sensory stimulation in vivo and indicate that low pCaMKII levels are associated with cutaneous mechanical hypersensitivity.

\section{AIP expression suppresses CaMKII activity}

To directly test whether loss of CaMKII feedback would produce excessive responsiveness to cutaneous mechanical stimuli in the intact animal, we used a genetic approach to inhibit CaMKII signaling in sensory neurons in vivo. Specifically, we used an AAV vector expressing AIP (Fig. 3A), a well-established peptide inhibitor of CaMKII activity derived from the regulatory domain of CaMKII (Ishida et al., 1995). This peptide inhibitor strategy blocks both the $\mathrm{Ca}^{2+}$ independent and-dependent forms of the kinase and all CaMKII isoforms. We targeted $\mathrm{A} \beta$-LTMRs by direct DRG injection (Fischer et al., 2011) of an AAV8 serotype vector that we have previously found to preferentially transduce large sensory neurons typical of this neuronal subgroup (Yu et al., 2013). The expressed EGFP-AIP fusion protein (Bok et al., 2007) is stable in transduced HEK293T cells (Fig. 3B). Transgene expression is present in sensory neurons within 1 week (Fischer et al., 2011; Yu et al., 2013), and vector injection into the L4 and L5 DRGs that innervate the plantar skin produces an overall transduction efficiency of $16 \pm 1 \%(N=6$ DRGs from 3 rats; Fig. $3 C, D)$. Higher transduction rates were seen in the medium/large neuron population (34 $\pm 3 \%$ in neurons with area $\left.700-1600 \mu \mathrm{m}^{2}\right)$ and in the largest neurons $(66 \pm 6 \%$ in neurons with area $>1600 \mu \mathrm{m}^{2}$; Fig. $4 A$ ). This tropism toward large neurons is consistent with observations by others (Jacques et al., 2012). Our findings thus suggest that we achieved significant genetic ablation of CaMKII signaling in neurons subserving touch.

Transduced neurons showed features of $\mathrm{A} \beta$-LTMRs, including large somatic size, and immunopositivity for neurofilament marker NF200 but minimal TrkA, CGRP, or IB4 staining (Fig. 4B). TrkA is only expressed in nociceptive sensory neurons, including all rapidly conducting A $\beta$ nociceptors (Fang et al., 2005). Here we observed TrkA expression in $8.6 \pm$ $2.4 \%$ of transduced neurons (4 fields in DRGs from 2 rats, total $n=152$ neurons), suggesting a minimal contribution of $\mathrm{A} \beta$ nociceptors to the behavioral changes observed after CaMKII blockade. Spinal cord projection to deeper dorsal horn laminae (Fig. $4 C-F$ ) is also typical of $\mathrm{A} \beta$ -
A
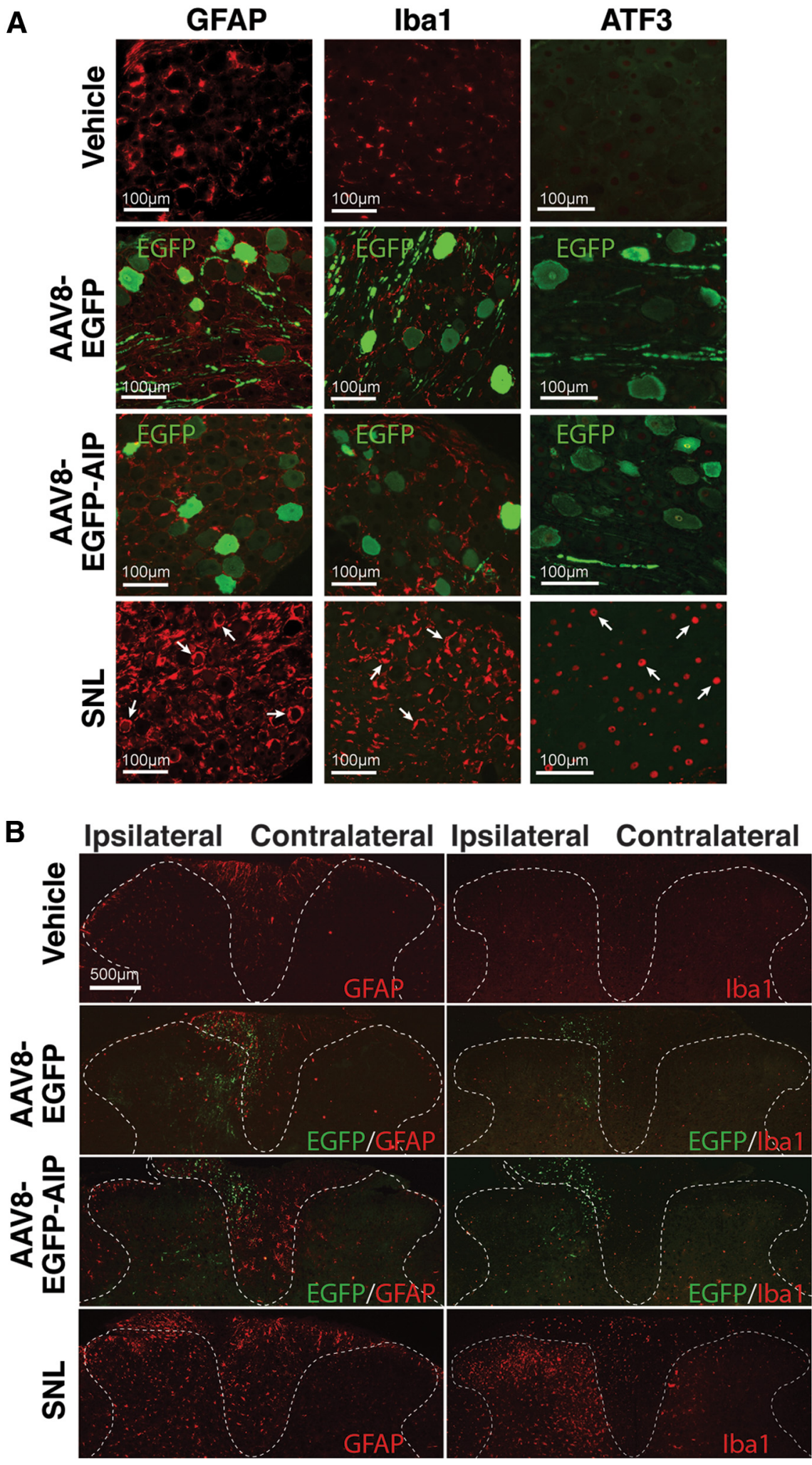

Figure 7. Neuroinflammation is absent following DRG vector injections. A, DRGL5 sections show immunostaining for GFAP (left column), lba1 (middle), and ATF3 (right) in DRG sections from rats 4 weeks after injection with vehicle (top row), AAV8-EGFP (second row), and AAV8-EGFP-AIP (third row), and from nerve-injured rats 4 weeks following L5 SNL (bottom row) used as a positive control. SNL markedly increased GFAP immunoreactivity bythickening rings of satellite glial cells (arrows), increased lba1 staining representing accumulation of residentmicroglia orhematogenous macrophages around neuronal somata (arrows), and increased ATF3 immunoreactivity in nudei of axotomized neurons (arrows). These changes wereabsentfollowing vehicleorvectorinjections. $n=3$ rats and 6 DRGsforeach condition. $B$,Spinal cord transversesections at L4/L5segmentallevel show immunostaining for GFAP (leftcolumn) and lba1 (right) from rats 4 weeks after L4/L5DRG injection with vehicle (top row), AAV8-EGFP (second row), and AAV8-EGFP-AIP (third row), and 4 weeks after SNL (bottom row). White dashed lines outline the dorsal horm. Injections did not increase GFAP or Iba1 immunoreactivity, whereas SNL triggers increased immunostaining for both. $n=3$ rats for each condition. 


\section{A von Frey}
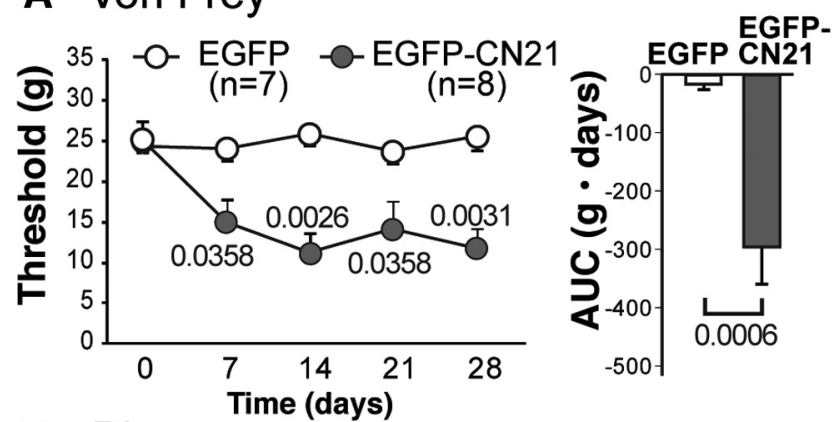

B Pin
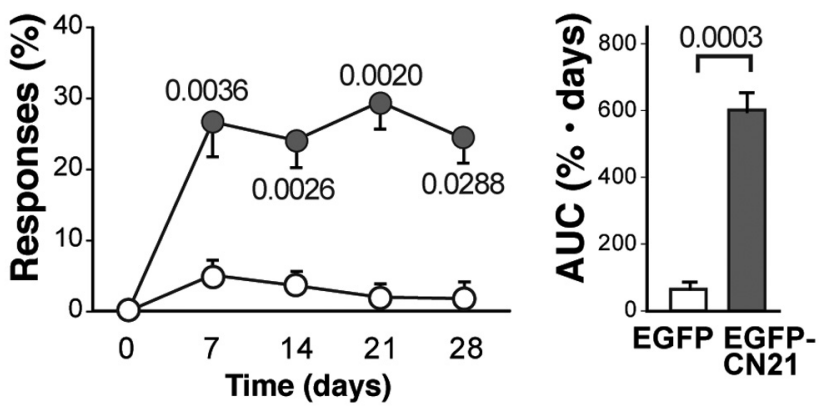

Figure 8. EGFP-CN21 expression in sensory neuron produces mechanosensory hypersensitivity. $\boldsymbol{A}$, Sensory function following ipsilateral vector injections into the $L 4$ and $L 5$ DRGs caused decreased threshold for withdrawal from touch (von Frey) after AAV6-EGFP-CN21 ( $p=0.0011$ ) but not for AAV6-EGFP ( $p=0.5944$ ). $p$ values are shown for significant paired comparisons to day $0 . B$, The frequency of complex, sustained hyperalgesia behavior after intense mechanical stimulation with a pin increased after AAV6-EGFP-CN21 ( $p=0.0016)$ but not for AAV6-EGFP $(p=0.4060)$. AUC for measures normalized to day 0 confirmed differences between groups for both measures. Data are mean \pm SEM.

LTMRs. Central processes of transduced neurons showed EGFP extending to presynaptic terminals, but dorsal horn neuronal somata showed no EGFP (Fig. 4D-H), indicating that transduction was limited to peripheral sensory neurons. EGFP-AIP was also found in peripheral processes of the transduced sensory neurons. Transduced fibers innervating the skin were almost exclusively associated with $A \beta$-LTMRs that terminated in Merkel endings (Fig. 5A) and Meissner corpuscles (Fig. 5B) in glabrous skin, as well as larger lanceolate endings in hairy skin (Fig. $5 C$ ). EGFP immunostaining was sometimes found at smaller lanceolate endings (Fig. $5 C$ ) that probably represent A $\delta$-LTMR units $(\mathrm{Li}$ et al., 2011), consistent with rare transduction of small/medium DRG neurons (Fig. 5D). No transduced free nerve endings typical of $A \delta$ or $C$ fiber nociceptors were identified. Although the smaller diameter of these fibers might make it more difficult to observe EGFP in them, we suspect that this is not the reason for their absence in our samples because such fibers are readily seen in transgenic mice expressing EGFP in sensory neurons (Zylka et al., 2005). Together, these findings indicate that transgene expression was limited to LTMRs and was present throughout their entire extent.

DRG neurons of all sizes showed immunostaining for pCaMKII (Fig. 5D), which was also present at the A $\beta$-LTMR peripheral terminals in skin tactile organs (Fig. $5 A-C$ ), suggesting that autophosphorylated CaMKII may be an essential control element in mechanosensation. To confirm that EGFP-AIP interferes with CaMKII signaling in transduced neurons, we measured pCaMKII immunofluorescence levels in DRG neuron cell bodies 4 weeks following vector injection (Fig. 5D). Although expression of EGFP alone had no effect, expression of EGFP-AIP diminished pCaMKII-immunoreactive level (Fig. 5E), indicating successful block of CaMKII activation and Thr 286/287 autophosphorylation in vivo.

\section{CaMKII blockade heightens mechanosensitivity}

The above results show that natural sensory stimulation activates CaMKII in A $\beta$-LTMRs. We next asked whether CaMKII activity regulates sensation conducted by A $\beta$-LTMRs. Sensory testing in rats injected with the control vector showed only mild temporary hypersensitivity (Fig. 6) comparable with that which develops in vehicle-injected rats (Fischer et al., 2011). In contrast, CaMKII blockade by EGFP-AIP expression in neurons innervating plantar skin produced hypersensitive mechanosensation tested both at low levels with von Frey fibers and with intense mechanical stimulation by pin, whereas dynamic touch, thermal sensitivity, and motor function were unaffected (Fig. 6). These behaviors represent aversive neuropathic pain (Hogan et al., 2004; Wu et al., 2010) and point to a key role for CaMKII in regulating $\mathrm{A} \beta$ LTMR responsiveness and potential contributions to pain.

Alternative explanations for aberrant sensory behavior following vector injection, such as inflammation, cellular immune reaction, or neurotoxicity in the DRG or spinal cord, were also considered. When we examined tissues from injected animals, we found no evidence that either vector produced neuronal injury, glial proliferation, microglial activation, or macrophage invasion in injected DRGs or associated spinal cord (Fig. 7). Nerve injury may induce a novel phenotype of mechanoreceptors, characterized by new expression of TRPV1 or CGRP (Rashid et al., 2003; Nitzan-Luques et al., 2011), but this was also not seen after vector injection (Fig. 4I). Thus, hyperalgesia in the animals injected with AAV8-EGFP-AIP can best be attributed to AIP block of CaMKII signaling in $A \beta$-LTMRs and consequent changes in their function.

To address the possibility that behavioral changes induced by CaMKII blockade were specific to the AAV8 vector or the AIP peptide, we prepared an AAV vector using the nonselective AAV6 serotype to express the unrelated CaMKII inhibitory peptide CN21 derived from the natural CaMKII inhibitor protein CaM-KIIN (Vest et al., 2007), which was linked to EGFP for imaging. Like the AIP inhibitor, expression of the CN21 peptide inhibitor of CaMKII also heightened sensitivity to mild and intense touch (Fig. 8), which shows that diverse CaMKII blockers produce pain behavior and that the necessary effect is on the A $\beta$-LTMRs. Together, these findings indicate that chronic CaMKII inhibition in A $\beta$-LTMRs is the cause of dramatic sensitization to mechanical stimulation, which results in a behavioral phenotype that closely replicates animal models of neuropathic pain (Hogan et al., 2004).

\section{CaMKII regulates adaptation and impulse propagation}

To identify the neuronal sites at which CaMKII might regulate A $\beta$-LTMR-mediated sensation, we first examined the process by which mechanical force is transduced into AP trains in sensory neuron peripheral terminals, and addressed the potential offtarget effects of our genetic inhibition of CaMKII by switching to a small membrane-permeable small-molecule inhibitor of CaMKII. Using an ex vivo skin-nerve preparation, we could directly evaluate AP train generation by sensory neurons subjected to CaMKII blockade during mechanical stimulation of the skin. In these experiments, we used the membrane-permeable CaMKII inhibitor KN-93 (Sumi et al., 1991) and the inactive analog KN92. We targeted $\mathrm{A} \beta$-LTMRs by recording mechanosensitive units 

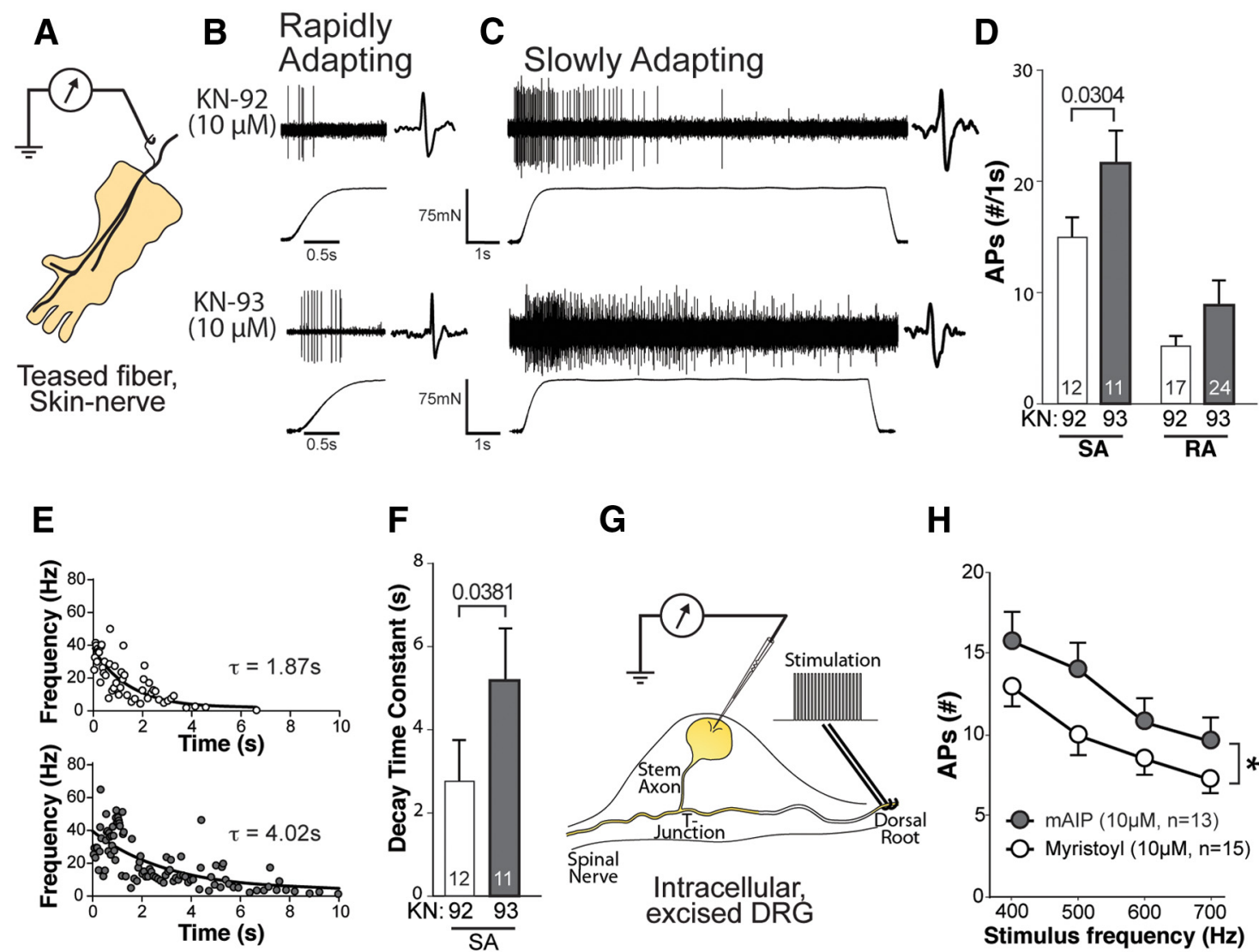

G

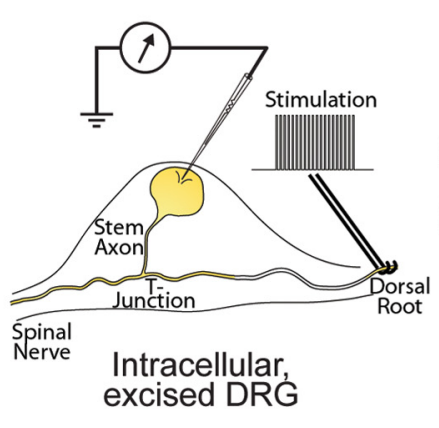

H

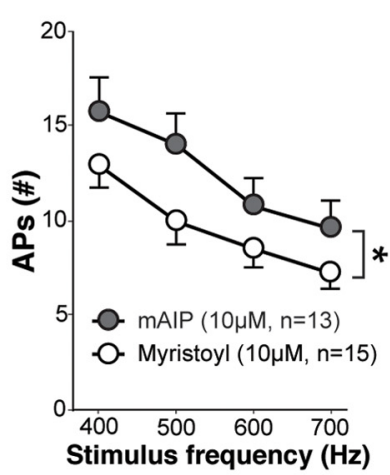

Figure 9. Effect of CaMKII blockade on impulse generation in skin and impulse propagation through DRGs. Using an ex vivo skin-sural nerve preparation $(\boldsymbol{A})$, sample traces $(\boldsymbol{B})$ illustrate AP generation by rapidly adapting units in response to mechanical stimulation (shown below trace) in the presence of the control compound KN-92 (top) and the CaMKII inhibitor KN-93 (bottom). Insets, Single-unit waveforms. C, Similar recordings are shown for slowly adapting units. $\boldsymbol{D}$, The number of APs in the first $1 \mathrm{~s}$ of stimulation is increased by KN-93 compared with KN-92 in slowly adapting $(S A)$ units, but not in rapidly adapting $(\mathrm{RA})$ units $(p=0.0984)$. $n$ is shown in each bar. $E$, Adaptation of firing rate was measured by fitting an exponential decay curve to the instantaneous frequency (1/[interspike interval] vs time data for each fiber). Top panel, From a unit treated with KN-92. Bottom panel, From a different unit treated with KN-93. $\boldsymbol{F}$, The time constant $\tau$ approximately doubled in neurons treated with KN-93 versus KN-92. When 20 axonal APs were triggered during intracellular recording in an excised, intact DRG preparation (G), the number that were successfully propagated to the soma was increased by mAIP $(\boldsymbol{H})$ compared with control (myristoyl); linear regression. ${ }^{*} p=0.0142$.

with $\mathrm{A} \beta$ conduction velocities $(>10 \mathrm{~m} / \mathrm{s})$ and either a slowly adapting or rapidly adapting AP train pattern typical of Merkel and Meissner units, respectively (Fig. 9A-C). Thresholds for firing were not affected by treatment (slowly adapting fibers: $4.7 \pm$ $1.0 \mathrm{mN}$ for $\mathrm{KN}-92,6.6 \pm 1.2 \mathrm{mN}$ for $\mathrm{KN}-93, p=0.24$; rapidly adapting fibers: $5.7 \pm 1.5 \mathrm{mN}$ for KN-92, $6.0 \pm 0.9 \mathrm{mN}$ for KN-93, $p=0.32$ ). To examine CaMKII regulation of the initial firing rate, we measured total APs in the first $1 \mathrm{~s}$ of mechanical stimulation, which revealed enhanced firing during CaMKII blockade, particularly in slowly adapting neurons (Fig. 9D). Impulse trains generated by $\mathrm{A} \beta$-LTMRs show adaptation in the form of progressively slower AP production during sustained skin stimulation (Kwan et al., 2009), so we examined whether this was due to a dynamic role of CaMKII activation during sustained repetitive firing. Because firing of rapidly adapting units terminated within $1 \mathrm{~s}$, analysis of firing patterns during sustained stimulation was not possible for these neurons. For slowly adapting units, we characterized adaptation by fitting an exponential decay curve to the plot of instantaneous frequency. CaMKII blockade led to prolongation of the decay constant $(\tau)$, indicating diminished adaptation (Fig. $9 E, F$ ). Thus, skin-nerve recordings demonstrate CaMKII participation in both tonic regulation of mechanically induced impulse generation, evident in the first second of activity, as well as in the progressive inhibition of excitability that accrues during sustained mechanical stimulation.
In addition to controlling impulse generation, we next examined whether CaMKII may also control the passage of afferent impulse traffic in $\mathrm{A} \beta$-LTMRs through axonal branch points, which are natural low-pass filters for AP trains (Gemes et al., 2013). To assay this, we measured the rate of AP propagation through the sensory neuron T-junction in an ex vivo DRG-dorsal root preparation by recording the arrival in the DRG cell body of APs conducted from a stimulation site in the dorsal root (Fig. $9 G$ ). This revealed significantly less filtering of AP trains (i.e., more APs were recorded in the soma) in rapidly conducting large sensory neurons during CaMKII blockade compared with control conditions (Fig. 9H). From these experiments, we infer that use-dependent filtering at sensory neuron branch points depends on the activity state of CaMKII and that sensory disuse may lead to excessive propagation of AP trains as they pass to the spinal cord.

To test whether these effects at the skin nerve terminals and axonal T-junctions are the result of CaMKII regulating intrinsic membrane properties, we additionally used two reduced models of excitability in dissociated sensory neuron somata. First, to replicate depolarizations at an impulse generation site, we examined whether CaMKII mediates the influence of prior membrane depolarization on subsequent AP generation (Fig. 10A-C). Repetitively firing neurons expressing only EGFP were less excitable following a conditioning pulse than at baseline, similar to non- 

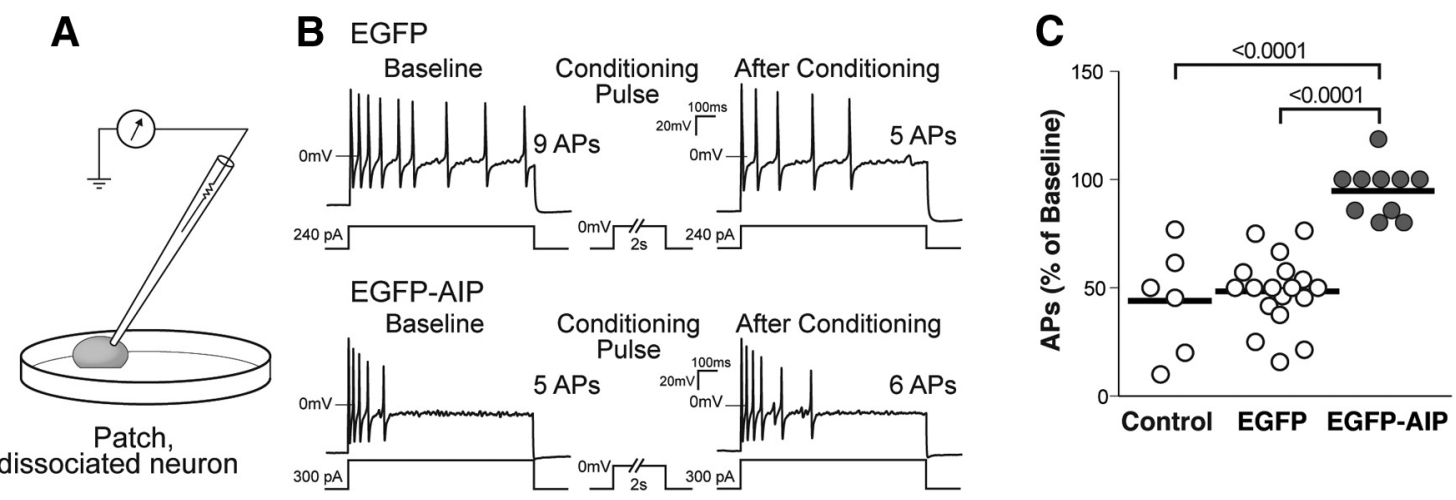

Figure 10. CaMKII controls neuronal excitability during impulse generation. Patch recording from dissociated sensory neurons $(\boldsymbol{A})$ showed that AP generation during current injection (1.5X rheobase) is reduced after conditioning ( $0 \mathrm{mV}$ for $2 \mathrm{~s}$ ) in neurons transduced by AAV8-EGFP but not after AAV8-EGFP-AIP $(\boldsymbol{B})$. C, Summary data show that firing is suppressed by prior neuronal activation in control (noninjected) neurons and in neurons expressing only EGFP, but this influence is eliminated in neurons expressing EGFP-AIP (ANOVA $p<0.0001$ ). Error bars indicate means.
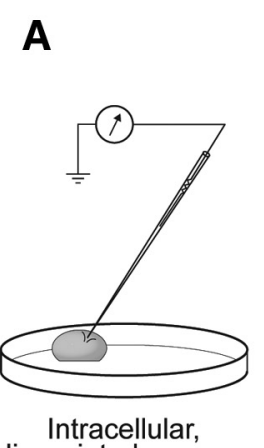
dissociated neuron
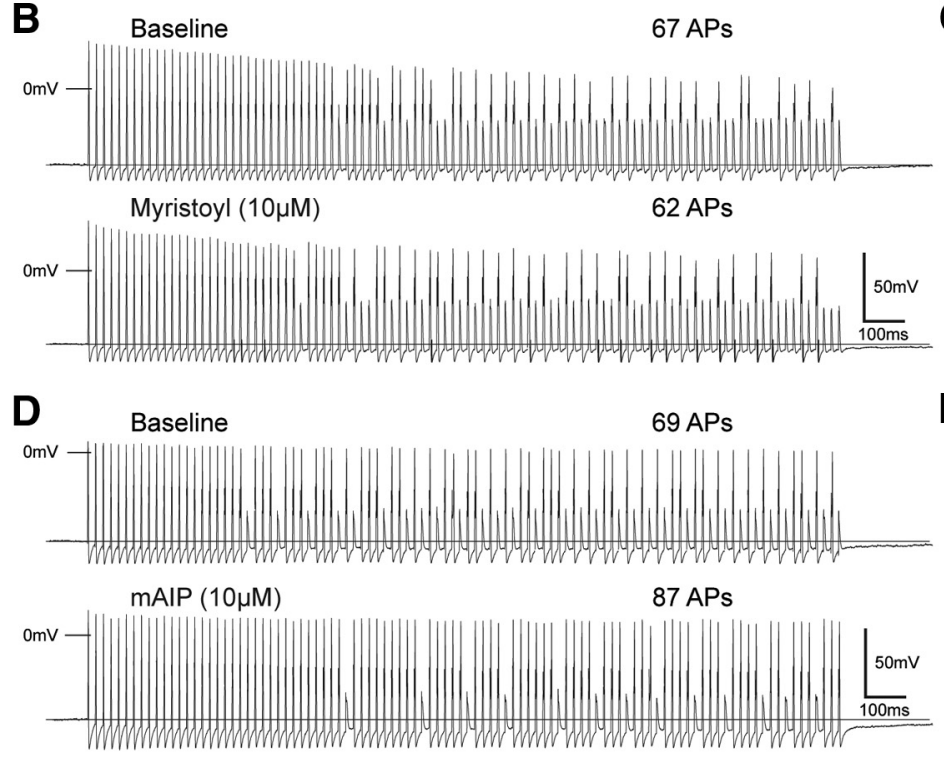

C

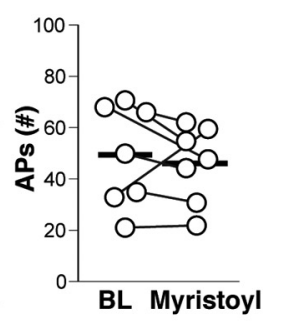

E

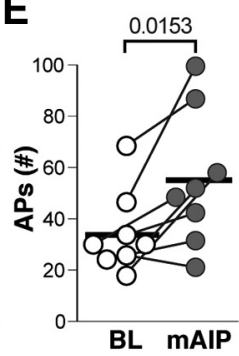

Figure 11. CaMKII regulates neuronal ability to follow repetitive stimulation. Intracellular recordings from dissociated sensory neurons $(A)$ during repetitive stimulation via current injection (1 ms pulse width, $1.5 \times$ rheobase, $50 \mathrm{~Hz}$ for $2 \mathrm{~s}$ ) show that the number of APs generated at baseline (BL) was not changed after 5 min application of myristoyl (B,C, $p=0.5883)$, whereas application of mAIP increased the number of $A P s(\boldsymbol{D}, \boldsymbol{E})$. Stimulus artifacts appear as small depolarizations. Neurons of the two groups did not differ in size or in resting $V_{M}, R_{i n}$, threshold, or number of $A P s$ under baseline conditions.

transduced control neurons. However, EGFP-AIP expression eliminated the influence of the conditioning pulse, causing AP generation to be insensitive to prior conditioning depolarization. This finding indicates that CaMKII provides an excitability brake that constrains AP generation based on previous neuronal activation.

To replicate membrane events during AP propagation through axonal branch points, we next examined CaMKII control of neuronal responsiveness to repetitive brief membrane depolarizations. To do so, we measured the number of APs that dissociated sensory neuron somata were able to generate during $50 \mathrm{~Hz}$ stimulation. This produces a characteristic pattern of progressively decreasing neuronal excitability during the tetanic stimulation (Fig. $11 A-C$ ), due to cytoplasmic $\mathrm{Ca}^{2+}$ accumulation (Gemes et al., 2010, 2013). CaMKII block increased the number of APs generated during the tetanic stimulation by reducing failures late in the train (Fig. $11 D, E$ ). Together, these data indicate that CaMKII controls membrane excitability in the contexts of both AP generation during sustained depolarization and in firing from brief repetitive depolarizations, supporting a critical role of CaMKII in the sensory terminals and T-junctions during mechanosensation.

\section{Mechanism of CaMKII control of excitability}

We next sought to identify the events downstream from CaMKII by which it regulates membrane excitability. Because full function of voltage-gated $\mathrm{Ca}^{2+}$ channels (VGCCs) in the membrane of sensory neurons depends on CaMKII signaling (Tang et al., 2012; Kostic et al., 2014), we speculated that CaMKII blockade enhances sensory neuron excitability through the loss of activityinduced cytoplasmic $\mathrm{Ca}^{2+}$ accumulation. We first confirmed an $\sim 30 \%$ reduction of the cytoplasmic $\mathrm{Ca}^{2+}$ rise during depolarization of neurons expressing EGFP-AIP (Fig. 12A-C). This is consistent with our previous data using small molecules and peptides to inhibit CaMKII regulation of VGCCs in vitro (Tang et al., 2012; Kostic et al., 2014). An expected downstream consequence would be diminished activation of $\mathrm{Ca}^{2+}$-activated $\mathrm{K}^{+}$channels $\left(\mathrm{K}_{(\mathrm{Ca})}\right.$ channels) (Swensen and Bean, 2003). Sensory neurons are criti- 
cally dependent on $\mathrm{K}_{(\mathrm{Ca})}$ channels for usedependent regulation of AP firing properties. These channels regulate membrane potential and can influence neuronal excitability on this basis, but $\mathrm{K}_{(\mathrm{Ca})}$ opening also reduces membrane $\mathrm{R}_{\mathrm{in}}$, which in turn limits the depolarization induced by an inward current (Sah, 1996; Scholz et al., 1998). We have previously noted that this control of $\mathrm{R}_{\mathrm{in}}$ is the dominant means by which $\mathrm{K}_{(\mathrm{Ca})}$ channels regulate sensory neuron excitability at the T-junction (Gemes et al., 2013). We therefore tested the dynamic control of $\mathrm{R}_{\mathrm{in}}$ in sensory neuron somata of intact DRGs in vitro after a train of APs initiated by axonal stimulation. Although AP trains substantially reduced $\mathrm{R}_{\text {in }}$ in control conditions, $\mathrm{R}_{\text {in }}$ was minimally suppressed during CaMKII blockade (Fig. 12D-F). Combined application of $\mathrm{K}_{(\mathrm{Ca})}$ blockers apamin and iberiotoxin had the same effect as CaMKII blockade and occluded any further effect of CaMKII blockade (Fig. 12G).

Together, our findings show that CaMKII responds to neuronal activity by dynamically regulating membrane excitability via control of $\mathrm{R}_{\mathrm{in}}$ at the level of the sensory neuron soma and also at axon sites, such as peripheral terminals and axonal branch points, which govern generation and propagation of AP trains. Our data support a novel mechanism for activity-dependent regulation of sensory neuron excitability (Fig. 12H) in which the degree of CaMKII activation encodes the neuronal firing history and then functions as a corrective feedback to regulate intrinsic neuronal excitability.

\section{CaMKII regulation of $\mathrm{A} \beta$-LTMRs in the dorsal horn}

Up to this point, we have shown that deficient CaMKII signaling leads to elevated afferent impulse traffic in A $\beta$-LTMRs. We next determined how this flow of sensory information interacts with the CNS to produce sensations. Although A $\beta$-LTMRs project without synapses to the brain via the dorsal columns to convey the sense of touch, they additionally give off branches that enter the spinal cord dorsal horn (Fig. $13 A$ ). These sensory neuron-spinal cord interactions are critical sites for modulating pain transmission (Latremoliere and Woolf, 2009) and form the basis for the gatecontrol theory introduced by Melzack and Wall (1965) 50 years ago. According to this model, A $\beta$-LTMR activity inhibits transmission of pain signals delivered to the cord by high-threshold nociceptive sensory neurons. However, recent evidence also demonstrates latent excitatory $\mathrm{A} \beta$-LTMR input to the dorsal horn lamina I projection neurons that convey pain to the brain (Takazawa and MacDermott, 2010). We therefore next examined for the action of $I_{K(\text { ( a })}$ on $R_{\text {in }}$.

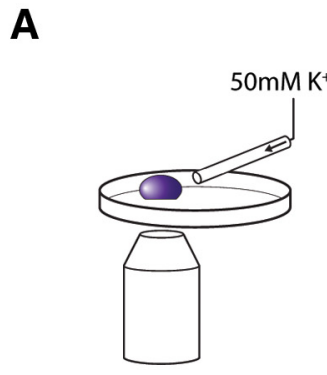

Fluorimetry, dissociated neuron

D

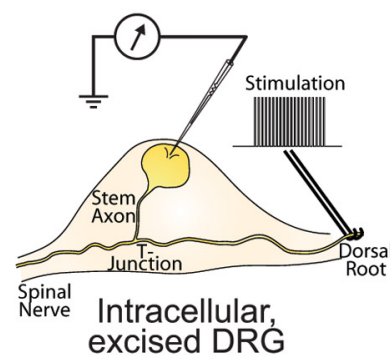

G

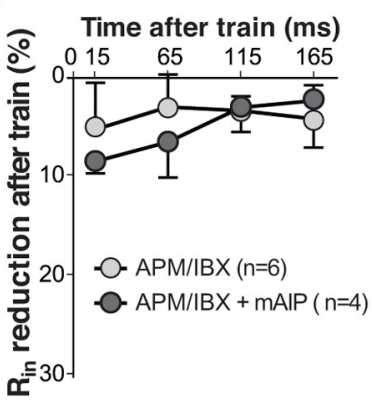

B

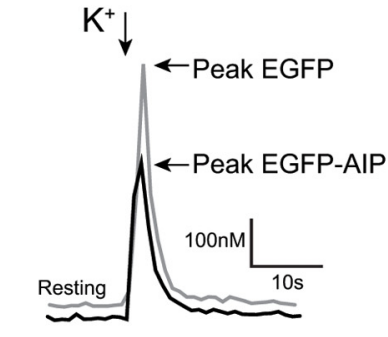

OnM
C

$\mathbf{F}$

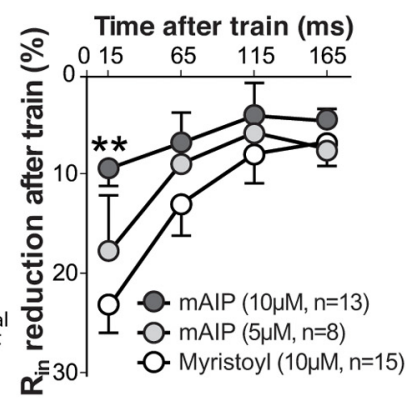

H

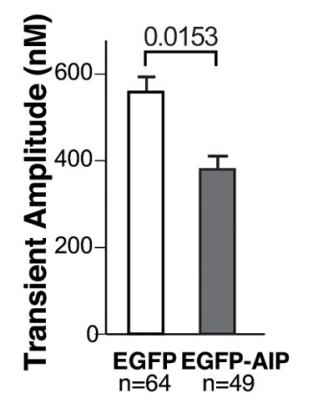

Figure 12. CaMKII controls membrane $R_{\text {in }}$ via $K_{(C a)}$ channels. Fluorimetric imaging of dissociated neurons $(\boldsymbol{A})$ revealed diminished amplitude (peak - resting level) of the cytoplasmic $\mathrm{Ca}^{2+}$ elevation induced by depolarization $\left(\boldsymbol{B}\right.$; arrow, $\left.\mathrm{K}^{+} 35 \mathrm{~mm}, 1 \mathrm{~s}\right)$ in neurons transduced by AAV8-EGFP-AIP compared with neurons transduced by AAV8-EGFP (C). Data are mean \pm SEM. ${ }^{* *} p<0.01$. During intracellular recording of neuronal $V_{M}$ in excised, intact DRGs $(\boldsymbol{D})$, membrane $R_{\text {in }}$ was determined at different intervals after the last AP of a train (20 APs per train, at each neuron's peak firing rate). $\boldsymbol{E}$, The post-train $R_{\text {in }}$ was reduced by mAIP: two-way repeated-measures ANOVA, main effects of agent $(p=0.0042)$, time $(p<0.0001)$, and interaction $(p<0.0001) .{ }^{* *} p=0.0012$ mAlP $10 \mu$ m versus myristoyl control (paired comparison). $F$, In other neurons, depression of $R_{\text {in }} 15 \mathrm{~ms}$ after an AP train was determined before and after agent application. Train-induced percentage $R_{\text {in }}$ reduction after agent was normalized against percentage reduction before agent. KN-93 substantially reduced train-induced depression of $\mathrm{R}_{\mathrm{in}}$, whereas the effect of $\mathrm{KN}-92 \mathrm{did}$ not differ from vehicle controls ( $0.2 \%$ DMSO in $n=5$, Tyrode's in $n=6)$; there was no difference, and these were combined. $p=$ 0.0003 (one-way ANOVA). G, Combined application of $\mathrm{K}_{(\mathrm{Ca})}$ channel blockers apamin (APM, $100 \mathrm{nM}$ ) and iberiotoxin (IBX, $100 \mathrm{nM}$ ) eliminated the effect of both a preceding AP train or the addition of mAIP $(10 \mu \mathrm{M})$ on $\mathrm{R}_{\text {in }}$ measured $15 \mathrm{~ms}$ after the last AP of the train: two-way repeated-measures ANOVA, main effects of agent $(p=0.6720)$, time $(p=0.5446)$, and interaction $(p=0.7154)$. $\boldsymbol{H}$, A control loop model for sensory adaptation in primary sensory neurons. All influences indicated by arrows are positive, except

whether CaMKII signaling affects the interactions of A $\beta$-LTMRs with this normally suppressed pain pathway. Recordings from lamina I neurons in tissue slices from animals with A $\beta$-LTMRs expressing EGFP-AIP demonstrated markedly elevated frequency of mEPSCs without any effect on their amplitude (Fig. $13 B-E)$. This indicates that loss of CaMKII signaling in $\mathrm{A} \beta$ LTMR enhances overall excitatory input to lamina I paintransmitting neurons. Blockade of inhibitory GABAergic and glycinergic synaptic activity with picrotoxin and strychnine 
A

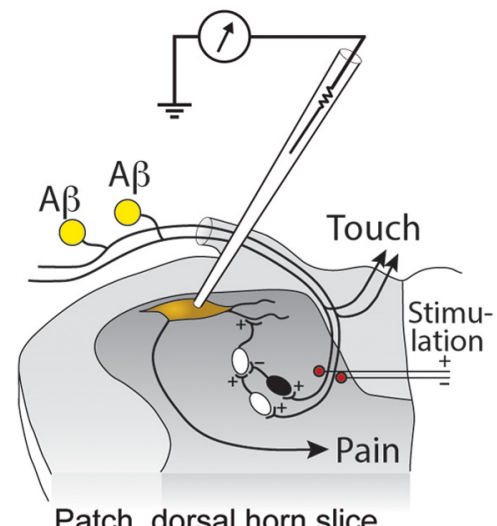

Patch, dorsal horn slice
B
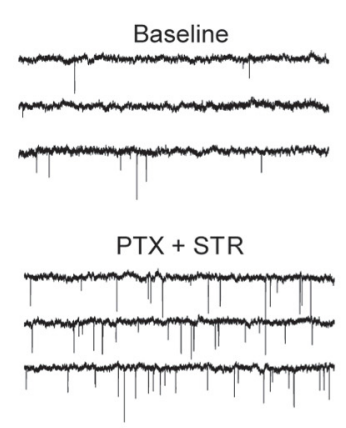

C

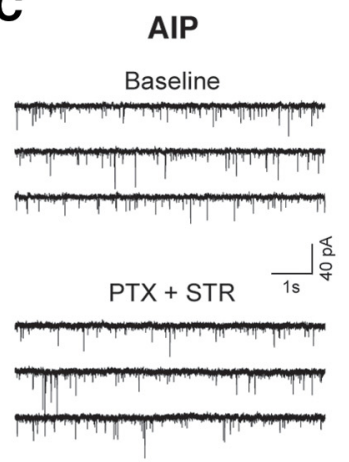

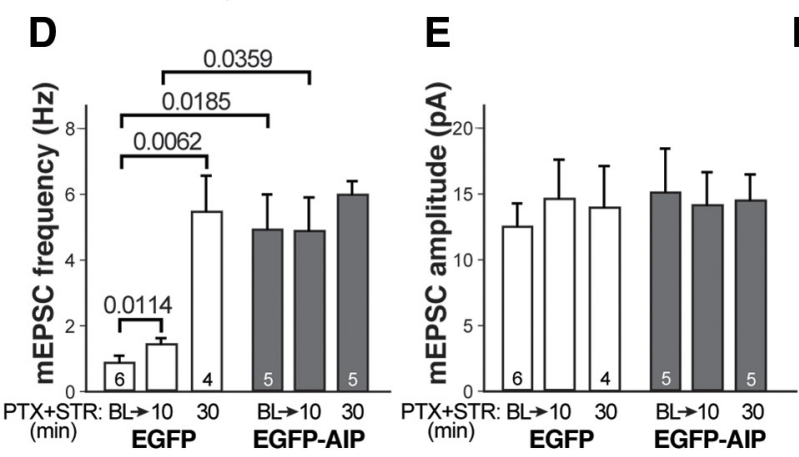

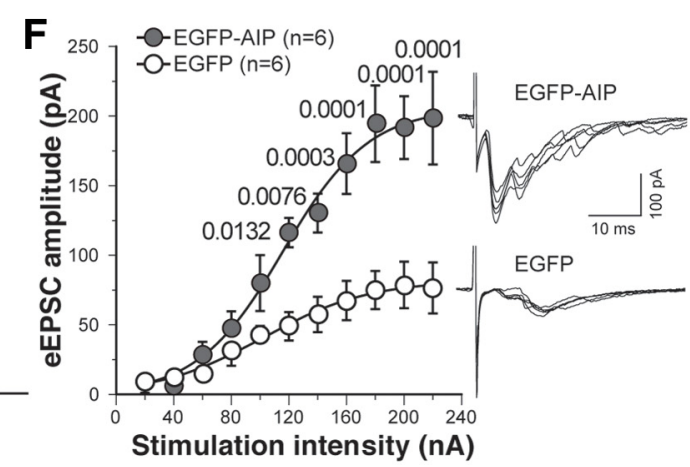

Figure 13. Influence of $A \beta$-LTMR CaMKII on dorsal horn network activation. $A$, Dorsal horn slice preparation, showing $A \beta$-LTMR terminating in deep laminae, interacting with a putative network of inhibitory (black) and excitatory (white) interneurons and lamina I projection neurons (brown). $\boldsymbol{B}, \mathrm{mEPSC}$ recorded in slices from animals with transduced sensory neurons expressing only EGFP show an increase in frequency when inhibitory influences are blocked by picrotoxin (PTX, $50 \mu \mathrm{M}$ ) and strychnine (STR, $5 \mu \mathrm{m}$ ) for $10 \mathrm{~min}$. C, Neurons expressing EGFP-AIP had elevated mEPSC frequency at baseline and were unresponsive to PTX and STR. D, Summary data, including additional neurons incubated in PTX + STR for 30 min, shows equalization of mEPSC frequency when neurons of both groups were exposed to PTX + STR. E, Amplitude of mEPSCs was not affected by transgene expression. $\boldsymbol{F}$, Evoked EPSCs in neurons expressing EGFP-AIP show greater amplitudes compared with neurons expressing EGFP alone, across a range of stimulus intensity $\left(p<0.001\right.$, main effect of group for two-way repeated-measures ANOVA). ${ }^{*} p<0.05$, compared with EGFP. Insets, eEPSC traces with typical polysynaptic patterns.

equalized mEPSC frequency by elevating the level in control slices without affecting recordings from EGFP-AIP-expressing animals. This makes clear that the inhibitory control to these lamina I neurons depends on A $\beta$-LTMR CaMKII. To directly test whether $A \beta$-LTMR input is a source of the disinhibited excitatory input, we evoked EPSCs by direct stimulation of A $\beta$-LTMR fibers (Fig. 13A). Slices from rats expressing only EGFP in A $\beta$ LTMRs developed no measurable current in 7 of 13 neurons, whereas all neurons from animals expressing EGFP-AIP $(n=6)$ showed evoked currents $(p<0.05)$. Additionally, considering only neurons that did respond to stimulation, EGFP-AIP expression by A $\beta$-LTMRs elevated the stimulus/amplitude relationship compared with recordings from EGFP-expressing animals (Fig. $13 F$ ), which confirms that EGFP-AIP in A $\beta$-LTMRs removed the inhibition of this dorsal horn network, permitting polysynaptic activation of nociceptive lamina I neurons by A $\beta$-LTMRs. Thus, in parallel with our findings that $A \beta$-LTMRs in the periphery require CaMKII to adjust AP generation and propagation according to past activity, these final findings reveal an additional mechanism at the level of the spinal cord whereby CaMKII signaling is critical for maintaining the inhibitory role of $\mathrm{A} \beta$ LTMRs.

\section{Discussion}

Here, we show that CaMKII regulates the flow of sensory information in A $\beta$-LTMR sensory neurons at multiple levels, including impulse generation, AP propagation, and dorsal horn synaptic transmission. At the peripheral terminals, CaMKII pro- vides a molecular substrate that accomplishes the fundamental requirement for sensory adaptation, which is the generation of APs inversely indexed to past neuronal activity. Although a shift in CaMKII activity will alter the state of numerous cytoplasmic and nuclear targets, our data are compatible with a simple model (Fig. $12 \mathrm{H}$ ) in which neuronal firing and cytoplasmic $\mathrm{Ca}^{2+}$ elevations are encoded by the conversion of CaMKII into its autophosphorylated form (pCaMKII), resulting in enhanced kinase function that boosts $\mathrm{Ca}^{2+}$ influx through VGCCs during membrane depolarization events, which in turn opens $\mathrm{Ca}^{2+}$-sensitive $\mathrm{K}^{+}$channels, by which $\mathrm{R}_{\text {in }}$ is depressed. This reduces excitability, which is the final inhibitory feedback control element for governing future AP generation. A component of this system is found in vestibular neurons (Nelson et al., 2005), where spontaneous firing rate is modulated by CaMKII control of current through $\mathrm{K}_{(\mathrm{Ca})}$ channels. However, unlike vestibular neurons, A $\beta$-LTMRs are naturally quiescent in the absence of mechanical stimulation of their receptive field. Our data suggest that A $\beta$-LTMRs need only periodic neuronal activation by natural use (e.g., whisking, ambulation) to support prolonged elevation of pCaMKII levels above those that are observed following sensory disuse. This role of CaMKII in the peripheral nervous system is in stark contrast to its role in the CNS discussed for the past 20 years, in which CaMKII is hypothesized to translate neuronal firing frequency into enhanced synaptic function.

We additionally observed that pain behavior develops when CaMKII function is selectively inhibited in the A $\beta$-LTMR popu- 
lation. These neurons normally produce the nonpainful sensation of touch, so our findings raise a fundamental question about the role $\mathrm{A} \beta$-LTMR neurons play in painful conditions. Although it is well established that activation of $\mathrm{A} \beta$-LTMRs can cause pain (Campbell et al., 1988), this phenomenon is most often seen following dorsal horn sensitization by prior conditioning via elevated C-fiber nociceptive activity, a process that was absent in our experiments. It has also been proposed that nerve injury may induce a phenotypic shift in A $\beta$-LTMRs, but this also was not evident in our study. Finally, a subpopulation of $\mathrm{A} \beta$ neurons naturally convey a nociceptive modality (Djouhri and Lawson, 2004). These units have central processes that project to the superficial dorsal horn (Light and Perl, 1979; Woodbury et al., 2008), express TrkA (Fang et al., 2005), and lack complex tactile organs at their peripheral terminals (Kruger et al., 1981), unlike our transduced $\mathrm{A} \beta$-LTMR population. However, we cannot exclude a contribution from A $\beta$ nociceptors. Support for the view that primary pathology in $\mathrm{A} \beta$-LTMRs may directly generate pain comes from observations that the painful conditions of diabetes and chronic regional pain syndrome are associated with hypertrophied and disordered A $\beta$-LTMR terminations (Albrecht et al., 2006; Pare et al., 2007). We are left with the conclusion that a loss of CaMKII signaling in otherwise normal A $\beta$-LTMRs is adequate to cause them to convey pain. Involvement of sensory neuron CaMKII in pain mechanisms is also suggested by our recent finding that painful nerve injury results in reduced CaMKII activity in the injured sensory neurons (Bangaru et al., 2015).

In the dorsal horn, our data support a model whereby a sufficient level of CaMKII activity in A $\beta$-LTMRs is required to maintain the inhibitory network that blocks $\mathrm{A} \beta$-LTMR input to nociceptive lamina I neurons. Therefore, in a CaMKII-deficient state, not only will A $\beta$-LTMRs convey greater afferent traffic from the periphery by elevated generation and propagation of impulse trains, but also the gate will be open for A $\beta$-LTMR impulses to access the pain pathway (Fig. 6A). This may be a potent substrate for $\mathrm{A} \beta$-LTMR-mediated pain behavior. There is growing recognition that disinhibition of this pathway contributes to chronic pain states in various models (Baba et al., 2003; Takazawa and MacDermott, 2010). Previously described forms of disinhibition involve loss of inhibitory interneurons or decreased response of projection neurons to inhibitory neurotransmission (Latremoliere and Woolf, 2009). Our data now show that deficient CaMKII signaling restricted to the A $\beta$-LTMR neuron itself is sufficient to depress the inhibitory control that limits $A \beta$ LTMR access to lamina I pain projection neurons.

We observed that selective functional modulation of $\mathrm{A} \beta$ LTMR units by the AAV8-EGFP-AIP vector reduced the threshold for a withdrawal response to von Frey fibers from the baseline value of $23 \mathrm{~g}$ to $\sim 5 \mathrm{~g}$. We note that this is still above the threshold for activating individual $\mathrm{A} \beta$-LTMRs in both humans and rodents (Edin et al., 1995; Garrison et al., 2012). Discordance such as this between behavioral mechanical thresholds and the threshold for individual units has been noted previously. For instance, knockout of the transient receptor potential canonical 1 (TRPC1) channel substantially reduces frequency of withdrawal from light touch in behavioral testing but has no effect on the threshold for initiating activity in $\mathrm{A} \beta$-LTMR units recorded individually from ex vivo skin fibers (Garrison et al., 2012). However, when forces above threshold are examined, reduced AP firing is observed in the TRPC1 knock-outs. The importance of suprathreshold firing properties is also apparent in animals that lack Piezo2 (Woo et al., 2014). Although low-intensity touch is decreased in these mice, single-unit recordings show no change in the threshold force for firing, whereas suprathreshold forces evoke reduced AP generation by $\mathrm{A} \beta$-LTMR in the mice lacking Piezo2. Finally, although the average threshold for activating all types of individual skin units in anesthetized rats is at or $<15 \mathrm{mN}$ ( $1.5 \mathrm{~g}$ ) (Boada et al., 2010), the threshold for withdrawal from mechanical stimulation in normal rats is far above that. Thus, it is likely that a critical firing rate must be achieved to trigger withdrawal, probably also incorporating spatial summation from the many fibers with overlapping receptive fields in the area of stimulation. We infer that the heightened behavioral responses after CaMKII blockade are due to accelerated AP generation and propagation during suprathreshold stimulation of A $\beta$-LTMR. We have also previously observed that manipulations of A $\beta$-LTMRs that either augment firing (Garrison et al., 2014) or decrease firing (Woo et al., 2014) are not associated with increased behavioral withdrawal from dynamic mechanical stimulation (brush), consistent with our findings here.

It would be reasonable to expect that amplified generation and propagation of impulses by $\mathrm{A} \beta$-LTMRs secondary to reduced CaMKII signaling should produce simply a more powerful and extensive sensation of touch rather than pain, and might even be expected to reduce pain experience by activating segmental and descending analgesic pathways. Yet we observed reduced threshold for withdrawal from von Frey fibers and an increased frequency of the complex, sustained hyperalgesia behavior that are hallmarks of various validated neuropathic pain models. This latter finding is particularly relevant as our previous work has demonstrated that hyperalgesia behavior is selectively associated with conditioned place avoidance (Wu et al., 2010) and thereby represents an aversive sensory experience. The explanation for this change in sensory modality comes from our data showing that CaMKII is critically necessary to maintain inhibition of the latent dorsal horn pathways that link $\mathrm{A} \beta$-LTMRs to nociceptive projection neurons. Thus, pain in conditions of low A $\beta$-LTMR CaMKII signaling are attributable to the combined effects of elevated impulse traffic and opened access of this traffic to nociceptive pathways.

We have shown that sensory disuse causes pain to be conveyed by pathways normally dedicated to touch. In a clinical setting, disuse of an affected body part is a common consequence of painful conditions, such as inflammatory processes or nerve injury. Sensitization caused by disuse will not only supplement the pain caused by the primary painful disorder, but also may outlast the recovery of the original cause of pain and become a selfmaintaining source of chronic pain. Our findings suggest that treatments focused on elevating sensory neuron CaMKII signaling, including natural neuronal activation, may have particular analgesic benefit in painful conditions that lead to limb disuse and hence to loss of adaptive signaling in the mechanosensory system.

\section{References}

Aftanas M, Zubek JP (1963) Long-term after-effects following isolation of a circumscribed area of the skin. Percept Mot Skills 17:867-870. CrossRef Medline

Albrecht PJ, Hines S, Eisenberg E, Pud D, Finlay DR, Connolly MK, Paré M, Davar G, Rice FL (2006) Pathologic alterations of cutaneous innervation and vasculature in affected limbs from patients with complex regional pain syndrome. Pain 120:244-266. CrossRef Medline

Baba H, Ji RR, Kohno T, Moore KA, Ataka T, Wakai A, Okamoto M, Woolf CJ (2003) Removal of GABAergic inhibition facilitates polysynaptic A fibermediated excitatory transmission to the superficial spinal dorsal horn. Mol Cell Neurosci 24:818-830. CrossRef Medline

Bangaru ML, Meng J, Kaiser DJ, Yu H, Fischer G, Hogan QH, Hudmon A (2015) Differential expression of CaMKII isoforms and overall kinase 
activity in rat dorsal root ganglia after injury. Neuroscience 300:116-127. CrossRef Medline

Bensmaïa SJ, Leung YY, Hsiao SS, Johnson KO (2005) Vibratory adaptation of cutaneous mechanoreceptive afferents. J Neurophysiol 94:3023-3036. CrossRef Medline

Boada MD, Houle TT, Eisenach JC, Ririe DG (2010) Differing neurophysiologic mechanosensory input from glabrous and hairy skin in juvenile rats. J Neurophysiol 104:3568-3575. CrossRef Medline

Bok J, Wang Q, Huang J, Green SH (2007) CaMKII and CaMKIV mediate distinct prosurvival signaling pathways in response to depolarization in neurons. Mol Cell Neurosci 36:13-26. CrossRef Medline

Campbell JN, Raja SN, Meyer RA, Mackinnon SE (1988) Myelinated afferents signal the hyperalgesia associated with nerve injury. Pain 32:89-94. CrossRef Medline

Carlton SM (2002) Localization of CaMKIIalpha in rat primary sensory neurons: increase in inflammation. Brain Res 947:252-259. CrossRef Medline

Chaplan SR, Bach FW, Pogrel JW, Chung JM, Yaksh TL (1994) Quantitative assessment of tactile allodynia in the rat paw. J Neurosci Methods 53: 55-63. CrossRef Medline

Djouhri L, Lawson SN (2004) Abeta-fiber nociceptive primary afferent neurons: a review of incidence and properties in relation to other afferent A-fiber neurons in mammals. Brain Res Brain Res Rev 46:131-145. CrossRef Medline

Duncan C, Mueller S, Simon E, Renger JJ, Uebele VN, Hogan QH, Wu HE (2013) Painful nerve injury decreases sarco-endoplasmic reticulum $\mathrm{Ca}(2)(+)$-ATPase activity in axotomized sensory neurons. Neuroscience 231:247-257. CrossRef Medline

Edin BB, Essick GK, Trulsson M, Olsson KA (1995) Receptor encoding of moving tactile stimuli in humans: I. Temporal pattern of discharge of individual low-threshold mechanoreceptors. J Neurosci 15:830-847. Medline

Fang X, Djouhri L, McMullan S, Berry C, Okuse K, Waxman SG, Lawson SN (2005) trkA is expressed in nociceptive neurons and influences electrophysiological properties via Nav1.8 expression in rapidly conducting nociceptors. J Neurosci 25:4868-4878. CrossRef Medline

Fischer G, Kostic S, Nakai H, Park F, Sapunar D, Yu H, Hogan Q (2011) Direct injection into the dorsal root ganglion: technical, behavioral, and histological observations. J Neurosci Methods 199:43-55. CrossRef Medline

Garrison SR, Dietrich A, Stucky CL (2012) TRPC1 contributes to lighttouch sensation and mechanical responses in low-threshold cutaneous sensory neurons. J Neurophysiol 107:913-922. CrossRef Medline

Garrison SR, Weyer AD, Barabas ME, Beutler BA, Stucky CL (2014) A gainof-function voltage-gated sodium channel 1.8 mutation drives intense hyperexcitability of A- and C-fiber neurons. Pain 155:896-905. CrossRef Medline

Gemes G, Rigaud M, Koopmeiners AS, Poroli MJ, Zoga V, Hogan QH (2010) Calcium signaling in intact dorsal root ganglia: new observations and the effect of injury. Anesthesiology 113:134-146. CrossRef Medline

Gemes G, Oyster KD, Pan B, Wu HE, Bangaru ML, Tang Q, Hogan QH (2012) Painful nerve injury increases plasma membrane $\mathrm{Ca}^{2+}$-ATPase activity in axotomized sensory neurons. Mol Pain 8:46. CrossRef Medline

Gemes G, Koopmeiners A, Rigaud M, Lirk P, Sapunar D, Bangaru ML, Vilceanu D, Garrison SR, Ljubkovic M, Mueller SJ, Stucky CL, Hogan QH (2013) Failure of action potential propagation in sensory neurons: mechanisms and loss of afferent filtering in C-type units after painful nerve injury. J Physiol 591:1111-1131. CrossRef Medline

Grynkiewicz G, Poenie M, Tsien RY (1985) A new generation of $\mathrm{Ca}^{2+}$ indicators with greatly improved fluorescence properties. J Biol Chem 260: 3440-3450. Medline

Guo TZ, Offley SC, Boyd EA, Jacobs CR, Kingery WS (2004) Substance P signaling contributes to the vascular and nociceptive abnormalities observed in a tibial fracture rat model of complex regional pain syndrome type I. Pain 108:95-107. CrossRef Medline

Hogan Q, Sapunar D, Modric-Jednacak K, McCallum JB (2004) Detection of neuropathic pain in a rat model of peripheral nerve injury. Anesthesiology 101:476-487. CrossRef Medline

Hudmon A, Schulman H (2002) Neuronal $\mathrm{CA}^{2+} /$ calmodulin-dependent protein kinase II: the role of structure and autoregulation in cellular function. Annu Rev Biochem 71:473-510. CrossRef Medline

Ishida A, Kameshita I, Okuno S, Kitani T, Fujisawa H (1995) A novel highly specific and potent inhibitor of calmodulin-dependent protein kinase II. Biochem Biophys Res Commun 212:806-812. CrossRef Medline

Jacques SJ, Ahmed Z, Forbes A, Douglas MR, Vigenswara V, Berry M, Logan A (2012) AAV8(gfp) preferentially targets large diameter dorsal root ganglion neurones after both intra-dorsal root ganglion and intrathecal injection. Mol Cell Neurosci 49:464-474. CrossRef Medline

Kojundzic SL, Puljak L, Hogan Q, Sapunar D (2010) Depression of Ca(2+)/ calmodulin-dependent protein kinase II in dorsal root ganglion neurons after spinal nerve ligation. J Comp Neurol 518:64-74. CrossRef Medline

Kostic S, Pan B, Guo Y, Yu H, Sapunar D, Kwok WM, Hudmon A, Wu HE, Hogan QH (2014) Regulation of voltage-gated Ca currents by $\mathrm{Ca} /$ calmodulin-dependent protein kinase II in resting sensory neurons. Mol Cell Neurosci 62C:10-18. CrossRef Medline

Kruger L, Perl ER, Sedivec MJ (1981) Fine structure of myelinated mechanical nociceptor endings in cat hairy skin. J Comp Neurol 198:137-154. CrossRef Medline

Kwan KY, Glazer JM, Corey DP, Rice FL, Stucky CL (2009) TRPA1 modulates mechanotransduction in cutaneous sensory neurons. J Neurosci 29: 4808-4819. CrossRef Medline

Latremoliere A, Woolf CJ (2009) Central sensitization: a generator of pain hypersensitivity by central neural plasticity. J Pain 10:895-926. CrossRef Medline

Li L, Rutlin M, Abraira VE, Cassidy C, Kus L, Gong S, Jankowski MP, Luo W, Heintz N, Koerber HR, Woodbury CJ, Ginty DD (2011) The functional organization of cutaneous low-threshold mechanosensory neurons. Cell 147:1615-1627. CrossRef Medline

Light AR, Perl ER (1979) Spinal termination of functionally identified primary afferent neurons with slowly conducting myelinated fibers. J Comp Neurol 186:133-150. CrossRef Medline

Loewenstein WR, Mendelson M (1965) Components of receptor adaptation in a Pacinian corpuscle. J Physiol 177:377-397. CrossRef Medline

Lüscher C, Streit J, Quadroni R, Lüscher HR (1994) Action potential propagation through embryonic dorsal root ganglion cells in culture: I. Influence of the cell morphology on propagation properties. J Neurophysiol 72:622-633. Medline

Melzack R, Wall PD (1965) Pain mechanisms: a new theory. Science 150: 971-979. CrossRef Medline

Morey-Holton ER, Globus RK (2002) Hindlimb unloading rodent model: technical aspects. J Appl Physiol 92:1367-1377. CrossRef Medline

Nelson AB, Gittis AH, du Lac S (2005) Decreases in CaMKII activity trigger persistent potentiation of intrinsic excitability in spontaneously firing vestibular nucleus neurons. Neuron 46:623-631. CrossRef Medline

Nitzan-Luques A, Devor M, Tal M (2011) Genotype-selective phenotypic switch in primary afferent neurons contributes to neuropathic pain. Pain 152:2413-2426. CrossRef Medline

Paré M, Albrecht PJ, Noto CJ, Bodkin NL, Pittenger GL, Schreyer DJ, Tigno XT, Hansen BC, Rice FL (2007) Differential hypertrophy and atrophy among all types of cutaneous innervation in the glabrous skin of the monkey hand during aging and naturally occurring type 2 diabetes. J Comp Neurol 501:543-567. CrossRef Medline

Rashid MH, Inoue M, Kondo S, Kawashima T, Bakoshi S, Ueda H (2003) Novel expression of vanilloid receptor 1 on capsaicin-insensitive fibers accounts for the analgesic effect of capsaicin cream in neuropathic pain. J Pharmacol Exp Ther 304:940-948. CrossRef Medline

Sah P (1996) $\mathrm{Ca}(2+)$-activated $\mathrm{K}^{+}$currents in neurones: types, physiological roles and modulation. Trends Neurosci 19:150-154. CrossRef Medline

Scholz A, Gruss M, Vogel W (1998) Properties and functions of calciumactivated $\mathrm{K}+$ channels in small neurones of rat dorsal root ganglion studied in a thin slice preparation. J Physiol 513:55-69. CrossRef Medline

Sumi M, Kiuchi K, Ishikawa T, Ishii A, Hagiwara M, Nagatsu T, Hidaka H (1991) The newly synthesized selective $\mathrm{Ca}^{2+} /$ calmodulin dependent protein kinase II inhibitor KN-93 reduces dopamine contents in PC12h cells. Biochem Biophys Res Commun 181:968-975. CrossRef Medline

Swensen AM, Bean BP (2003) Ionic mechanisms of burst firing in dissociated Purkinje neurons. J Neurosci 23:9650-9663. Medline

Takazawa T, MacDermott AB (2010) Synaptic pathways and inhibitory gates in the spinal cord dorsal horn. Ann N Y Acad Sci 1198:153-158. CrossRef Medline

Tang Q, Bangaru ML, Kostic S, Pan B, Wu HE, Koopmeiners AS, Yu H, Fischer GJ, McCallum JB, Kwok WM, Hudmon A, Hogan QH (2012) $\mathrm{Ca}(2)(+)$-dependent regulation of $\mathrm{Ca}(2)(+)$ currents in rat primary af- 
ferent neurons: role of CaMKII and the effect of injury. J Neurosci 32: 11737-11749. CrossRef Medline

Terkelsen AJ, Bach FW, Jensen TS (2008) Experimental forearm immobilization in humans induces cold and mechanical hyperalgesia. Anesthesiology 109:297-307. CrossRef Medline

Torre V, Ashmore JF, Lamb TD, Menini A (1995) Transduction and adaptation in sensory receptor cells. J Neurosci 15:7757-7768. Medline

Vest RS, Davies KD, O'Leary H, Port JD, Bayer KU (2007) Dual mechanism of a natural CaMKII inhibitor. Mol Biol Cell 18:5024-5033. CrossRef Medline

Webster MA (2012) Evolving concepts of sensory adaptation. F1000 Biol Rep 4:21. CrossRef Medline

Woo SH, Ranade S, Weyer AD, Dubin AE, Baba Y, Qiu Z, Petrus M, Miyamoto T, Reddy K, Lumpkin EA, Stucky CL, Patapoutian A (2014) Piezo2 is required for Merkel-cell mechanotransduction. Nature 509: 622-626. CrossRef Medline
Woodbury CJ, Kullmann FA, McIlwrath SL, Koerber HR (2008) Identity of myelinated cutaneous sensory neurons projecting to nocireceptive laminae following nerve injury in adult mice. J Comp Neurol 508:500-509. CrossRef Medline

Wu HE, Gemes G, Zoga V, Kawano T, Hogan QH (2010) Learned avoidance from noxious mechanical simulation but not threshold semmes weinstein filament stimulation after nerve injury in rats. J Pain 11:280286. CrossRef Medline

Yu H, Fischer G, Ferhatovic L, Fan F, Light AR, Weihrauch D, Sapunar D, Nakai H, Park F, Hogan QH (2013) Intraganglionic AAV6 results in efficient and long-term gene transfer to peripheral sensory nervous system in adult rats. PLoS One 8:e61266. CrossRef Medline

Zylka MJ, Rice FL, Anderson DJ (2005) Topographically distinct epidermal nociceptive circuits revealed by axonal tracers targeted to Mrgprd. Neuron 45:17-25. CrossRef Medline 